\title{
Subdomain-based flux-free a posteriori error estimators *
}

\author{
Núria PARÉS, Pedro DÍEZ and Antonio HUERTA* \\ Laboratori de Càlcul Numèric (www-lacan.upc.es), Departament de Matemàtica \\ Aplicada III, Universitat Politècnica de Catalunya, Barcelona, SPAIN.
}

\begin{abstract}
A new residual-type flux-free error estimator is presented. It computes upper and lower bounds of the error in energy norm with the ultimate goal of obtaining bounds for outputs of interest. The proposed approach precludes the main drawbacks of standard residual type estimators circumventing the need of flux-equilibration and resulting in a simple implementation that uses standard resources available in finite element codes. This is especially interesting for existing codes and 3D applications where the implementation of this technique is as simple as in 2D. Recall that on the contrary, the complexity of the flux-equilibration techniques increases drastically in the 3D case. Bounds for the energy norm of the error are used to produce upper and lower bounds of linear functional outputs, representing quantities of engineering interest. This new flux-free error estimator improves the effectivity of previous approaches (better accuracy in every test) and it can be used in the mechanical case for linear elements. The proposed approach demonstrates its efficiency in numerical tests producing sharp bounds of the reference error both for the energy and the quantities of interest.
\end{abstract}

Key words: Error estimation, error bounds, functional outputs, engineering outputs, goal-oriented error estimation, residual based estimators

\footnotetext{
* Sponsored by Ministerio de Ciencia y Tecnología (grants: DPI2004-3000 and CGL2004-06171-C03-01) and the Generalitat de Catalunya (grant: 2001SGR00257)

* Correspondence to: Antonio Huerta, Departament de Matemàtica Aplicada III, E.T.S. de Ingenieros de Caminos, Canales y Puertos, Universitat Politècnica de Catalunya, Jordi Girona 1, E-08034 Barcelona, SPAIN. Email address: antonio.huerta@upc.es (Antonio HUERTA).
} 


\section{Introduction}

Assessment of functional outputs of the solution (goal-oriented error estimation) in computational mechanics problems is a real need in standard engineering practice. In particular, end-users of finite element codes are interested in obtaining bounds for quantities of engineering interest. Techniques providing these bounds require using error estimators in the energy norm of the solution. Bounds for quantities of interest (functional outputs) are recovered combining upper and lower bounds of the energy error for both the original problem (primal) and a dual problem (associated with the selected functional output) [1-3].

It is also important to note that bounds for the energy and for quantities of interest are usually obtained with respect to a reference solution (associated with a much richer discretization, viz. a finer mesh). Bounds for the exact solution of the boundary value problem as presented in [4-6] are not addressed

\section{here.}

The need of obtaining reliable upper and lower bounds of the error for quantities of interest has motivated the use of residual error estimators, which are currently the oniy type of estimators ensuring bounds for the arror. Classical residual type estimators, which provide upper bounds of the er or, require
flux-equilibration procedures (hybrid-flux techniques) to properly set bound-
ary conditions for local problems $[7,2]$. Flux-equilibration is performed by a
complex algorithm, strong y dependent on the element type and requiring a data structure that is not natural in a standard finite element code.

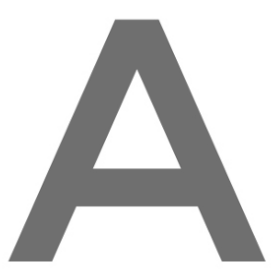

Register for free at https//www.scipedia.com to download the version without the watermark The idea of using flux-free estimators, based on the partition-o1-the-unity concept and using local subdomains different than elements, has been already proposed in [8-10]. The main advantage of the flux-free approach is the simplicity in the implementation. Obviously, this is especially important in the 3D case. Boundary conditions of the local problems are trivial and the usual data structure of a finite element code is directly employed. Recently, in [11], the flux-free estimates have been compared with the standard hybrid-flux estimates in terms of both their sharpness (effectivity) and their computational efficiency. The main conclusion of this investigation is that in most of the test cases the hybrid-flux estimates are more accurate while the overall computational cost is lower for the flux-free estimates. However, there is no general consensus on these issues.

This paper introduces a new flux-free error estimator improving the effectivity of previous approaches (better accuracy in every test), which can be used in the mechanical case for linear elements and with a further simplification in the implementation. The remainder of the paper is structured as follows. In 
Section 2, the model problem is described. The development of this technique is motivated by the need of assessing and bounding the error of the functional outputs of the solution. Then, in Section 3, a procedure to obtain upper and lower bounds of the energy norm is presented. Section 4 is devoted to analyze the features of the proposed estimates, including proofs of the main properties. In Section 5 the approach introduced here is compared with previously published flux-free techniques. The energy norm estimates are used in Section 6 to assess the error in quantities of interest. Computational aspects of the proposed methodology and some implementation details are discussed in Section 7. Finally, in Section 8, the different estimators are used in four numerical examples, from a simple 2D thermal problem to a $3 \mathrm{D}$ mechanical test.

\section{Statement of the problem}

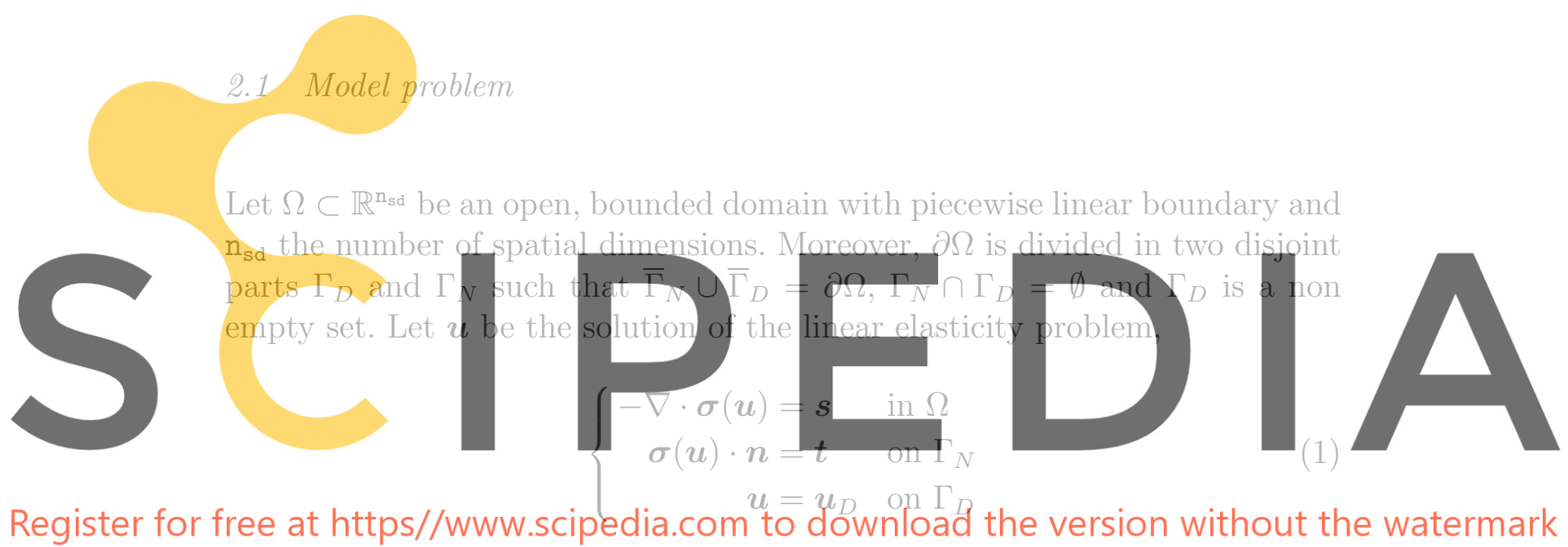

where $t$ and $u_{D}$ are the imposed traction and boundary displacements, respectively.

The weak solution of this problem is $\boldsymbol{u} \in \mathcal{U}$ verifying

$$
a(\boldsymbol{u}, \boldsymbol{v})=l(\boldsymbol{v}) \quad \forall \boldsymbol{v} \in \mathcal{V}
$$

where

$$
a(\boldsymbol{u}, \boldsymbol{v})=\int_{\Omega} \boldsymbol{\sigma}(\boldsymbol{u}): \boldsymbol{\varepsilon}(\boldsymbol{v}) d \Omega, \quad l(\boldsymbol{v})=\int_{\Omega} \boldsymbol{s} \cdot \boldsymbol{v} d \Omega+\int_{\Gamma_{N}} \boldsymbol{t} \cdot \boldsymbol{v} d \Gamma .
$$

The usual solution and test spaces are defined $\boldsymbol{U}=\left\{\boldsymbol{u} \in\left[\mathcal{H}^{1}(\Omega)\right]^{\mathrm{n}_{\mathrm{sd}}},\left.\boldsymbol{u}\right|_{\Gamma_{D}}=\right.$ $\left.\boldsymbol{u}_{D}\right\}$ and $\mathcal{V}=\left\{\boldsymbol{v} \in\left[\mathcal{H}^{1}(\Omega)\right]^{\mathrm{n}_{\mathrm{sd}}},\left.\boldsymbol{v}\right|_{\Gamma_{D}}=0\right\}$, where $\mathcal{H}^{1}$ is the standard Sobolev space of square integrable functions and first derivatives. The bilinear form $a(\cdot, \cdot)$ induces the energy norm, which is denoted by $\|\cdot\|$, that is, $\|\boldsymbol{v}\|^{2}=$ $a(\boldsymbol{v}, \boldsymbol{v})$. 
The finite element interpolation spaces $\mathcal{U}^{H} \subset \mathcal{U}$ and $\mathcal{V}^{H} \subset \mathcal{V}$ are associated with a finite element mesh of characteristic size $H$ and degree $p$ for the complete interpolation polynomial base. The geometric support of the elements for a given mesh are open subdomains denoted by $\Omega_{k}, k=1 \ldots \mathrm{n}_{\mathrm{e} 1}$, where $\bar{\Omega}=\bigcup_{k} \bar{\Omega}_{k}$. It is also assumed that different elements do not overlap, that is, $\Omega_{k} \cap \Omega_{l}=\emptyset$ for $k \neq l$.

Then, the finite element solution $\boldsymbol{u}_{H}$ which is an approximation to $\boldsymbol{u}$, lies in the finite dimensional space $\mathcal{U}^{H}$ and verifies

$$
a\left(\boldsymbol{u}_{H}, \boldsymbol{v}\right)=l(\boldsymbol{v}) \quad \forall \boldsymbol{v} \in \mathcal{V}^{H}
$$

\subsection{Error equations and reference error}

The goal of a posteriori error estimation is to assess the accuracy of the finite element solution $\boldsymbol{u}_{H}$, that is, to evaluate and measure the error, $\boldsymbol{e}:=\boldsymbol{u}-\boldsymbol{u}_{H}$, which belongs to $\mathcal{V}$, either in the energy norm $\|e\|$ or in a quantity of interest $l^{\mathcal{O}}(e)$.
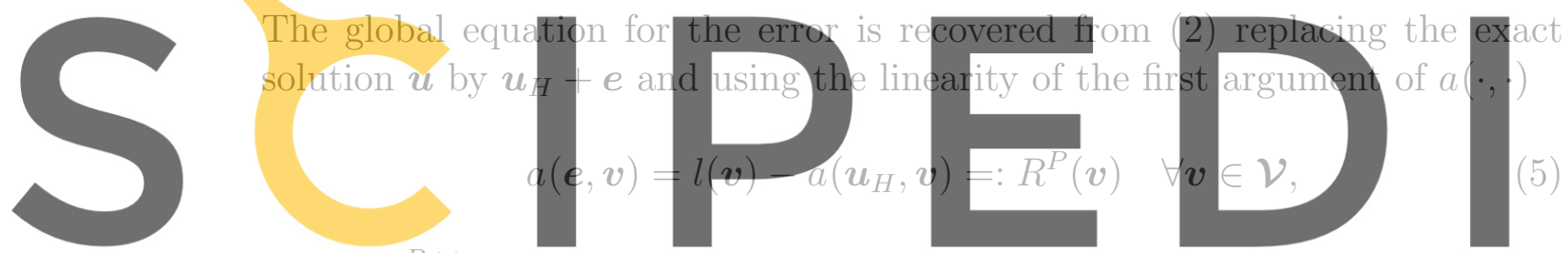

where $R^{P}(\cdot)$ stands for the weak residue associated to the finite element ap-

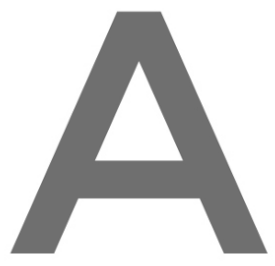

Register for fpeexift

In practice, the exact error $e$ is replaced by a reference error, $e_{h}$, lying in a finite dimensional space $\mathcal{V}^{h}$ much richer than the original finite element space $\mathcal{V}^{H}$. That is, the exact solution $\boldsymbol{u}$ is replaced by the reference (or truth) solution $\boldsymbol{u}_{h}$; consequently, $\boldsymbol{u} \approx \boldsymbol{u}_{h}=\boldsymbol{u}_{H}+\boldsymbol{e}_{h}$. The reference error is the projection of the exact error into the reference space, that is, $\boldsymbol{e}_{h} \in \mathcal{V}^{h}$ is the solution of the problem

$$
a\left(\boldsymbol{e}_{h}, \boldsymbol{v}\right)=R^{P}(\boldsymbol{v}) \quad \forall \boldsymbol{v} \in \mathcal{V}^{h}
$$

The direct computation of $\boldsymbol{e}_{h}$ is computationally unaffordable because the size of the system of equations is the dimension of $\mathcal{V}^{h}$. The idea behind any implicit residual type error estimator is to solve a set of local problems instead of the global problem (6). In each of these local problems, boundary conditions must be properly defined in order to obtain a good approximation of the error and to ensure solvability. 


\subsection{Estimation of outputs of interest}

Attention is usually centered in bounding output quantities $l^{\mathcal{O}}(\boldsymbol{u})$, where $l^{\mathcal{O}}(\cdot)$ is a linear functional, see for instance $[1,12,13,3,14]$. These strategies introduce a dual (or adjoint) problem with respect to the selected output. The weak form of the dual problem reads: find $\boldsymbol{\psi} \in \mathcal{V}$ such that

$$
a(\boldsymbol{v}, \boldsymbol{\psi})=l^{\mathcal{O}}(\boldsymbol{v}) \quad \forall \boldsymbol{v} \in \mathcal{V}
$$

The finite element approximation of the dual problem is $\boldsymbol{\psi}_{H} \in \mathcal{V}^{H}$ such that

$$
a\left(\boldsymbol{v}, \boldsymbol{\psi}_{H}\right)=l^{\mathcal{O}}(\boldsymbol{v}) \quad \forall \boldsymbol{v} \in \mathcal{V}^{H}
$$

Finally, the dual reference error is $\boldsymbol{\epsilon}_{h} \in \mathcal{V}^{h}$, such that

$$
a\left(v, \epsilon_{h}\right)=l^{\mathcal{O}}(v)-a\left(v, \psi_{H}\right)=: R^{D}(v) \quad \forall v \in \mathcal{v}^{h},
$$

where $R^{D}$ is the weak residue associated with $\psi_{H}$.

If $v$ is replaced by $\boldsymbol{e}_{h}$ in (8), then using Galerkin orthogonality and the parallelogram identity, the following representation of $l^{\mathcal{O}}\left(\boldsymbol{e}_{h}\right)$ can be obtained
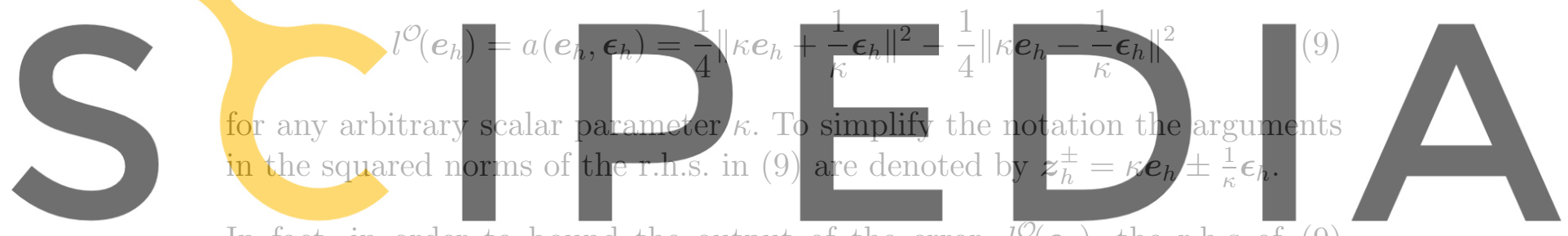

In fact, in order to bound the output of the error, $l^{O}\left(e_{h}\right)$, the r.h.s of $(9)$

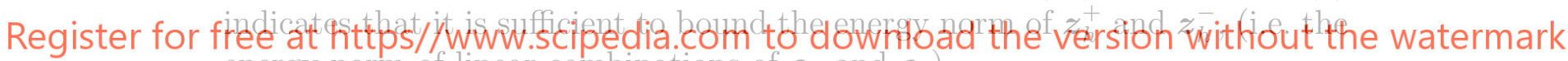
energy norm of linear combinations of $e_{h}$ and $\epsilon_{h}$ ).

Define $\mathrm{E}_{\mathrm{u}}[\boldsymbol{v}]$ and $\mathrm{E}_{\mathrm{l}}[\boldsymbol{v}]$ as the upper and lower bound of $\|\boldsymbol{v}\|^{2}$, respectively. Note that $\mathrm{E}_{\mathrm{u}}[\boldsymbol{v}]$ and $\mathrm{E}_{\mathrm{l}}[\boldsymbol{v}]$ are not functions; instead, it is a convenient notation of the upper and lower bounds of $\|\boldsymbol{v}\|^{2}$. Thus, once the bounds for $\left\|\boldsymbol{z}_{h}^{ \pm}\right\|^{2}$ are computed, namely

$$
\mathrm{E}_{\mathrm{l}}\left[\boldsymbol{z}_{h}^{ \pm}\right] \leq\left\|\boldsymbol{z}_{h}^{ \pm}\right\|^{2} \leq \mathrm{E}_{\mathrm{u}}\left[\boldsymbol{z}_{h}^{ \pm}\right]
$$

the output of the error is readily bounded as

$$
\frac{1}{4} \mathrm{E}_{\mathrm{l}}\left[\boldsymbol{z}_{h}^{+}\right]-\frac{1}{4} \mathrm{E}_{\mathrm{u}}\left[\boldsymbol{z}_{h}^{-}\right] \leq l^{\mathcal{O}}\left(\boldsymbol{e}_{h}\right) \leq \frac{1}{4} \mathrm{E}_{\mathrm{u}}\left[\boldsymbol{z}_{h}^{+}\right]-\frac{1}{4} \mathrm{E}_{\mathrm{l}}\left[\boldsymbol{z}_{h}^{-}\right] .
$$

This procedure is summarized in Figure 1 where bounds for the output of interest of the reference approximation, $l^{\mathcal{O}}\left(\boldsymbol{u}_{h}\right)$, are also shown: $l^{\mathcal{O}}\left(\boldsymbol{u}_{H}\right)$ is added to each term of inequality (10). Next section introduces a methodology to obtain both upper and lower bound error estimates in energy norm. This approach is then used to compute $\mathrm{E}_{\mathrm{u}}\left[\boldsymbol{z}_{h}^{+}\right], \mathrm{E}_{\mathrm{u}}\left[\boldsymbol{z}_{h}^{-}\right], \mathrm{E}_{\mathrm{l}}\left[\boldsymbol{z}_{h}^{+}\right]$and $\mathrm{E}_{\mathrm{l}}\left[\boldsymbol{z}_{h}^{-}\right]$. 
Step 1.- Compute $\boldsymbol{u}_{H} \in \mathcal{U}^{H}$.

Step 2.- Compute $\boldsymbol{\psi}_{H} \in \mathcal{V}^{H}$.

Step 3.- Compute $\mathrm{E}_{\mathrm{u}}\left[\boldsymbol{z}_{h}^{+}\right]$and $\mathrm{E}_{\mathrm{u}}\left[\boldsymbol{z}_{h}^{-}\right]$such that

$$
\left\|\boldsymbol{z}_{h}^{+}\right\|^{2} \leq \mathrm{E}_{\mathrm{u}}\left[\boldsymbol{z}_{h}^{+}\right] \quad \text { and } \quad\left\|\boldsymbol{z}_{h}^{-}\right\|^{2} \leq \mathrm{E}_{\mathrm{u}}\left[\boldsymbol{z}_{h}^{-}\right] .
$$

Step 4.- Compute $\mathrm{E}_{\mathrm{l}}\left[\boldsymbol{z}_{h}^{+}\right]$and $\mathrm{E}_{\mathrm{l}}\left[\boldsymbol{z}_{h}^{-}\right]$such that

$$
\mathrm{E}_{\mathrm{l}}\left[\boldsymbol{z}_{h}^{+}\right] \leq\left\|\boldsymbol{z}_{h}^{+}\right\|^{2} \quad \text { and } \quad \mathrm{E}_{\mathrm{l}}\left[\boldsymbol{z}_{h}^{-}\right] \leq\left\|\boldsymbol{z}_{h}^{-}\right\|^{2} .
$$

Step 5.- Compute bounds for the error in the quantity of interest as

$$
\frac{1}{4} \mathrm{E}_{\mathrm{l}}\left[\boldsymbol{z}_{h}^{+}\right]-\frac{1}{4} \mathrm{E}_{\mathrm{u}}\left[\boldsymbol{z}_{h}^{-}\right] \leq l^{\mathcal{O}}\left(\boldsymbol{e}_{h}\right) \leq \frac{1}{4} \mathrm{E}_{\mathrm{u}}\left[\boldsymbol{z}_{h}^{+}\right]-\frac{1}{4} \mathrm{E}_{\mathrm{l}}\left[\boldsymbol{z}_{h}^{-}\right] .
$$

Step 6.- Recover bounds for the output of interest as

$$
\begin{aligned}
& l^{\mathcal{O}}\left(\boldsymbol{u}_{H}\right)+\frac{1}{4} \mathrm{E}_{\mathrm{u}}\left[\boldsymbol{z}_{h}^{+}\right]-\frac{1}{4} \mathrm{E}_{\mathrm{l}}\left[\boldsymbol{z}_{h}^{-}\right] \geq l^{\mathcal{O}}\left(\boldsymbol{u}_{h}\right), \\
& l^{\mathcal{O}}\left(\boldsymbol{u}_{H}\right)+\frac{1}{4} \mathrm{E}_{\mathrm{l}}\left[\boldsymbol{z}_{h}^{+}\right]-\frac{1}{4} \mathrm{E}_{\mathrm{u}}\left[\boldsymbol{z}_{h}^{-}\right] \leq l^{\mathcal{O}}\left(\boldsymbol{u}_{h}\right) .
\end{aligned}
$$
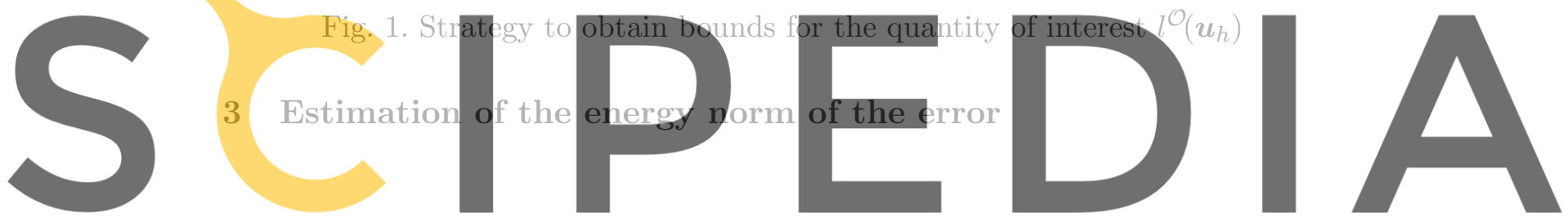

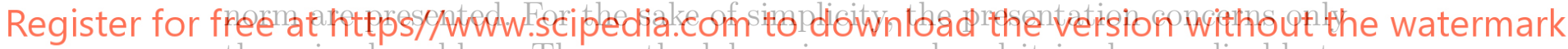
the primal problem. The methodology is general and it is also applicable to the dual problem or to linear combinations of both.

\subsection{Definitions and preliminaries}

Let $\boldsymbol{x}^{i}, i=1, \ldots, \mathrm{n}_{\mathrm{np}}$ denote the vertices of the elements in the computational mesh (thus linked to $\mathcal{U}^{H}$ ) and $\phi^{i}$ the corresponding linear (or bilinear or trilinear) shape functions, which are such that $\phi^{i}\left(\boldsymbol{x}^{j}\right)=\delta_{i j}$. The support of $\phi^{i}$ is denoted by $\omega^{i}$ and it is called the star centered in, or associated with, vertex $\boldsymbol{x}^{i}$.

It is important to recall that the linear shape functions based on the vertices are a partition of unity. Using this essential property and the linearity of the weak residue $R^{P}(\cdot)$, defined in (5), for every $\boldsymbol{v} \in\left[\mathcal{H}^{1}(\Omega)\right]^{\mathrm{n}_{\mathrm{sd}}}$ the following 
equality holds

$$
R^{P}(\boldsymbol{v})=R^{P}\left(\sum_{i=1}^{\mathrm{n}_{\mathrm{np}}} \phi^{i} \boldsymbol{v}\right)=\sum_{i=1}^{\mathrm{n}_{\mathrm{np}}} R^{P}\left(\phi^{i} \boldsymbol{v}\right) .
$$

Note that $R^{P}\left(\phi^{i} \boldsymbol{v}\right)$ vanishes if $\operatorname{supp} \boldsymbol{v} \cap \omega^{i}=\emptyset$, because $\omega^{i}$ is the support of $\phi^{i}$. Therefore, the residue is decomposed into local contributions over each star. This basic property is the key idea to define residual estimators based in stars. Similar approaches have been used in references [15,8-10].

Let $\mathcal{V}_{\omega^{i}}^{h}$ and $\mathcal{V}_{\omega^{i}}^{H}$ denote the local restrictions of the reference and finite element spaces to the star $\omega^{i}$, that is,

$$
\mathcal{V}_{\omega^{i}}^{h}:=\mathcal{V}^{h} \cap\left[\mathcal{H}^{1}\left(\omega^{i}\right)\right]^{\mathrm{n}_{\mathrm{sd}}} \quad \text { and } \quad \mathcal{V}_{\omega^{i}}^{H}:=\mathcal{V}^{H} \cap\left[\mathcal{H}^{1}\left(\omega^{i}\right)\right]^{\mathrm{n}_{\mathrm{sd}}}
$$

Formally any function $v \in \mathcal{V}_{\omega^{i}}^{h}$ (in particular, $v \in \mathcal{V}_{\omega^{i}}^{H} \subset \mathcal{V}_{\omega^{i}}^{h}$ ) is not defined in the whole domain $\Omega$ but only in the star $\omega^{i}$. However, here any $v \in \mathcal{V}_{\omega^{i}}^{h}$ is naturally extended to $\Omega$ by setting the values outside $\omega^{i}$ to zero. Thus, functions in $\mathcal{V}_{\omega^{i}}^{h}$ are continuous in $\omega^{i}$ but generally discontinuous across the boundary of the star $\omega^{i}$.
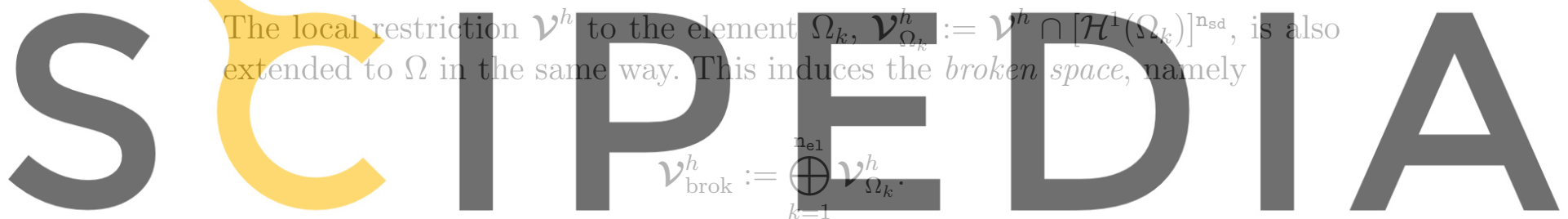

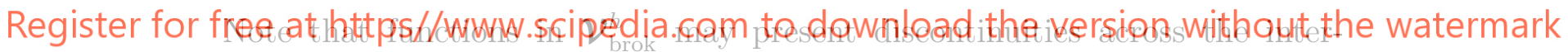
element edges (or faces) and that $\mathcal{V}_{\omega^{i}{ }^{h}} \subset \mathcal{V}_{\text {brok }}^{h}$.

The bilinear form $a(\cdot, \cdot)$ and the energy norm are generalized to accept broken functions in its arguments; that is, for $\boldsymbol{v}$ and $\boldsymbol{w} \in \mathcal{V}_{\text {brok }}^{h}$,

$$
a(\boldsymbol{v}, \boldsymbol{w}):=\sum_{k=1}^{\mathrm{n}_{\mathrm{e} 1}} a_{\Omega_{k}}(\boldsymbol{v}, \boldsymbol{w}) \quad \text { and } \quad\|\boldsymbol{v}\|^{2}:=\sum_{k=1}^{\mathrm{n}_{\mathrm{e} 1}}\|\boldsymbol{v}\|_{k}^{2}
$$

where $a_{\Omega_{k}}(\cdot, \cdot)$ is the restriction of the bilinear form $a(\cdot, \cdot)$ to the element $\Omega_{k}$ and $\|\boldsymbol{v}\|_{k}^{2}=a_{\Omega_{k}}(\boldsymbol{v}, \boldsymbol{v})$.

For further developments it is also necessary to introduce the nodal projections of any function in $\mathcal{V}$ onto the finite element space, $\mathcal{V}^{H}$, and the reference space, $\mathcal{V}^{h}$. That is, $\pi^{H}: \mathcal{V} \longrightarrow \mathcal{V}^{H}$ such that $\pi^{H} \boldsymbol{v}\left(\hat{\boldsymbol{x}}^{i}\right)=\boldsymbol{v}\left(\hat{\boldsymbol{x}}^{i}\right)$ where $\hat{\boldsymbol{x}}^{i}$ denote every node on the finite element mesh, and $\pi^{h}: \mathcal{V} \longrightarrow \mathcal{V}^{h}$ such that $\pi^{h} \boldsymbol{v}\left(\hat{\boldsymbol{x}}^{i}\right)=\boldsymbol{v}\left(\hat{\boldsymbol{x}}^{i}\right)$ where $\hat{\boldsymbol{x}}^{i}$ denote now the nodal points of the reference mesh. 
Step 1.- Compute $\boldsymbol{u}_{H} \in \mathcal{U}^{H}$.

Step 2.- For $i=1, \ldots, \mathrm{n}_{\mathrm{np}}$, compute $\tilde{\boldsymbol{e}}^{\omega^{i}} \in \mathcal{V}_{\omega^{i}}^{h}$ such that

$$
a_{\omega^{i}}\left(\tilde{\boldsymbol{e}}^{\omega^{i}}, \boldsymbol{v}\right)=R^{P}\left(\phi^{i}\left(\boldsymbol{v}-\boldsymbol{\pi}^{H} \boldsymbol{v}\right)\right) \quad \forall \boldsymbol{v} \in \mathcal{V}_{\omega^{i}}^{h}
$$

Step 3.- Define the global estimate $\tilde{\boldsymbol{e}} \in \mathcal{V}_{\text {brok }}^{h}$

$$
\tilde{\boldsymbol{e}}:=\sum_{i=1}^{\mathrm{n}_{\mathrm{np}}} \tilde{\boldsymbol{e}}^{\omega^{i}}
$$

Step 4.- Compute the upper bound as

$$
\mathrm{E}_{\mathrm{u}}\left[\boldsymbol{e}_{h}\right]=\|\tilde{\boldsymbol{e}}\|^{2}=\sum_{k=1}^{\mathrm{n}_{\mathrm{e} 1}}\|\tilde{\boldsymbol{e}}\|_{k}^{2} \geq\left\|\boldsymbol{e}_{h}\right\|^{2}
$$

Fig. 2. Upper bound for the squared energy norm of the reference error

Upper bound estimate of the reference error

The strategy to compute upper bound estimates of the reference error, $\mathrm{E}_{\mathrm{u}}\left[\boldsymbol{e}_{h}\right]$,
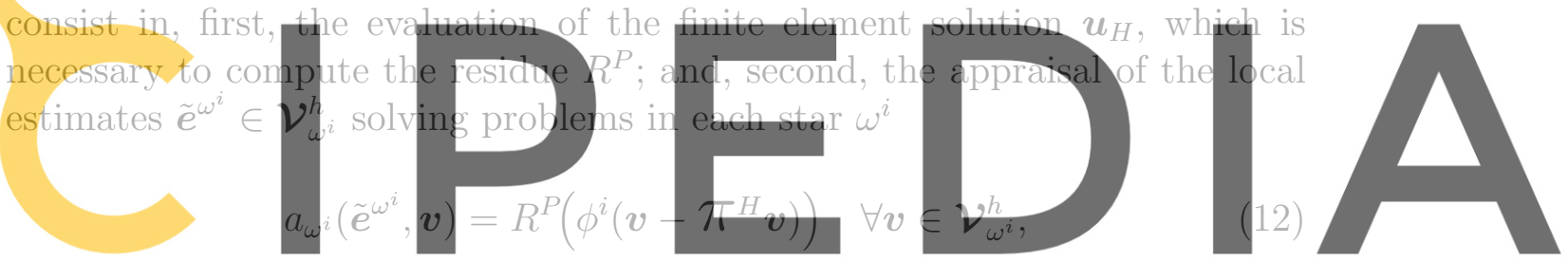

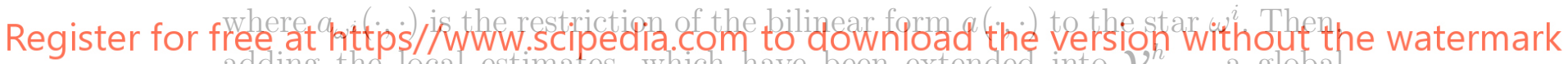
adding the local estimates, which have been extended into $\mathcal{V}_{\text {brok, a global }}$ estimate $\tilde{e} \in \mathcal{V}_{\text {brok }}^{h}$ is obtained,

$$
\tilde{\boldsymbol{e}}:=\sum_{i=1}^{\mathrm{n}_{\mathrm{np}}} \tilde{\boldsymbol{e}}^{\omega^{i}}
$$

and the upper bound of the energy norm of the reference error is recovered computing the norm of the estimate $\tilde{\boldsymbol{e}}$, that is, $\mathrm{E}_{\mathrm{u}}\left[\boldsymbol{e}_{h}\right]:=\|\tilde{\boldsymbol{e}}\|^{2} \geq\left\|\boldsymbol{e}_{h}\right\|^{2}$. Figure 2 describes this strategy in four steps.

Note that the error estimator described above does not require any computation of fluxes (stresses) along the boundary of the elements (it is flux-free).

Remark 1 In the r.h.s. of (12) the projection $\pi^{H}$ has been introduced in order to equilibrate the local problem and ensure its solvability. This is analyzed in Section 4.1. However, for scalar problems and mechanical problems with high-order elements (at least quadratic) the r.h.s. does not require the 
projection. That is, equation (12) reduces to

$$
a_{\omega^{i}}\left(\tilde{\boldsymbol{e}}^{\omega^{i}}, \boldsymbol{v}\right)=R^{P}\left(\phi^{i} \boldsymbol{v}\right) \quad \forall \boldsymbol{v} \in \mathcal{V}_{\omega^{i}}^{h}
$$

Remark 2 In Section 7 another expression for the r.h.s. of (12) is proposed to drastically simplify the practical implementation of this estimator.

\subsection{Lower bound estimates}

The upper bound estimate of the squared energy norm, $\mathrm{E}_{\mathrm{u}}\left[\boldsymbol{e}_{h}\right]$, is associated with the estimate $\tilde{\boldsymbol{e}}$ of the error function. The upper bound property is intrinsically related with the broken (discontinuous) nature of $\tilde{\boldsymbol{e}}$. On the contrary, a lower bound estimate is easily recovered from a continuous estimate of the error function, see [16]. Thus, once $\tilde{e}$ is obtained, a continuous estimate of the error function, $\tilde{e}_{\text {cont }}$, is computed by simple postprocessing. Two different alternatives can be considered to compute $\tilde{e}_{\text {cont }}$ from $\tilde{e}$. First, the strategy presented in detail in [16] and valid for any discontinuous estimate $\tilde{e}$ (discontinuous across inter-element edges or faces) can be readily implemented. It averages the discontinuities of the function across inter-element edges/faces
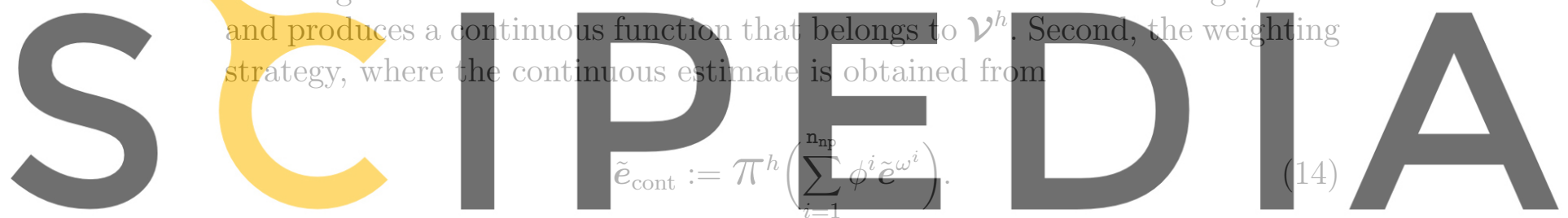

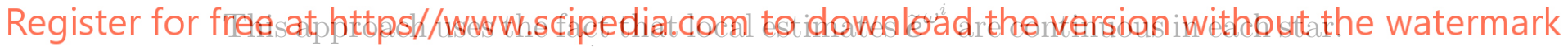

The discontinuities of $\tilde{e}^{\omega^{i}}$ on the boundary of each star $\omega^{i}$ are smoothed by multiplying by $\phi^{i}$, which vanishes along the boundary of $\omega^{i}$. Consequently, this is the natural choice for the estimates presented in this paper. The projection into the reference mesh $\mathcal{V}^{h}$ ensures that the evaluation of $R^{P}\left(\tilde{\boldsymbol{e}}_{\text {cont }}\right)$ is easily performed. Note that $\phi^{i} \tilde{\boldsymbol{e}}^{\omega^{i}}$ may not belong to $\mathcal{V}^{h}$

For both averaging strategies, a lower bound, $\mathrm{E}_{\mathrm{l}}\left[\boldsymbol{e}_{h}\right]$, of the energy norm of the reference error is obtained from $\tilde{\boldsymbol{e}}_{\text {cont }}$ as

$$
\mathrm{E}_{\mathrm{l}}\left[\boldsymbol{e}_{h}\right]:=\frac{\left(R^{P}\left(\tilde{\boldsymbol{e}}_{\text {cont }}\right)\right)^{2}}{\left\|\tilde{\boldsymbol{e}}_{\text {cont }}\right\|^{2}} \leq\left\|\boldsymbol{e}_{h}\right\|^{2} .
$$

Moreover, in order to improve the quality of the estimate the global enhancement strategy proposed in [17] can be implemented. First, $\tilde{\boldsymbol{e}}^{\mathrm{G}} \in \mathcal{V}^{H}$ is computed solving

$$
a\left(\tilde{\boldsymbol{e}}^{\mathrm{G}}, \boldsymbol{v}\right)=-a\left(\tilde{\boldsymbol{e}}_{\mathrm{cont}}, \boldsymbol{v}\right) \quad \forall \boldsymbol{v} \in \mathcal{V}^{H}
$$


Step 1.- Compute $\tilde{\boldsymbol{e}}_{\text {cont }} \in \mathcal{V}$ as

$$
\tilde{\boldsymbol{e}}_{\mathrm{cont}}=\pi^{h}\left(\sum_{i=1}^{\mathrm{n}_{\mathrm{np}}} \phi^{i} \tilde{\boldsymbol{e}}^{\omega^{i}}\right) .
$$

Step 2.- Compute the lower bound

$$
\mathrm{E}_{\mathrm{l}}\left[\boldsymbol{e}_{h}\right]:=\frac{\left(R^{P}\left(\tilde{\boldsymbol{e}}_{\mathrm{cont}}\right)\right)^{2}}{\left\|\tilde{\boldsymbol{e}}_{\mathrm{cont}}\right\|^{2}} \leq\left\|\boldsymbol{e}_{h}\right\|^{2} .
$$

Step 3.- Compute $\tilde{\boldsymbol{e}}^{\mathrm{G}} \in \mathcal{V}^{H}$ such that

$$
a\left(\tilde{\boldsymbol{e}}^{\mathrm{G}}, \boldsymbol{v}\right)=-a\left(\tilde{\boldsymbol{e}}_{\mathrm{cont}}, \boldsymbol{v}\right) \quad \forall \boldsymbol{v} \in \mathcal{V}^{H}
$$

Step 4.- Improve the lower bound as

$$
\mathrm{E}_{l}^{\mathrm{G}}\left[\boldsymbol{e}_{h}\right]:=\frac{\left(R^{P}\left(\tilde{\boldsymbol{e}}_{\mathrm{cont}}\right)\right)^{2}}{\left\|\tilde{\boldsymbol{e}}_{\text {cont }}\right\|^{2}-\left\|\tilde{\boldsymbol{e}}^{\mathrm{G}}\right\|^{2}} \leq\left\|\boldsymbol{e}_{h}\right\|^{2} .
$$

Fig. 3. Lower bounds for the squared energy norm of the error and then, the lower bound given in (15) is improved using $\left\|\tilde{e}^{\mathrm{G}}\right\|^{2}$ as
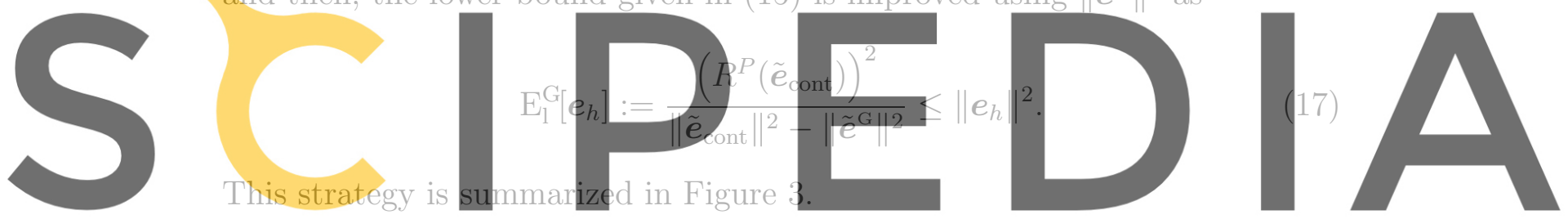

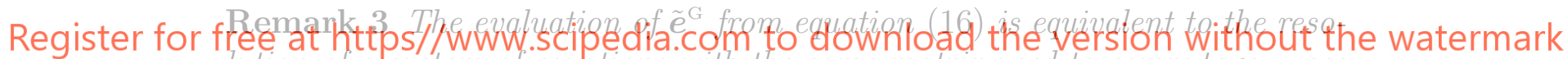
lution of a system of equations with the same matrix used to compute $\boldsymbol{u}_{H}$, see equation (4).

\section{Analysis and properties of the proposed estimates}

The upper bound estimate $\mathrm{E}_{\mathrm{u}}\left[\boldsymbol{e}_{h}\right]$ is obtained without any flux recovery or flux splitting technique. The effect of the flux jumps across each edge of the mesh is implicitly taken into account because the support of the local problems are the stars, which include the inter-element edges/faces. There is no need to compute and postprocess fluxes of the finite element solution, $\boldsymbol{u}_{H}$, along the inter-element edges/faces. Thus, the proposed estimate has two very attractive features:

(1) there is no need to compute fluxes and flux jumps along the element boundaries, and 
(2) there is no need to perform any flux equilibration.

Consequently, it is especially well suited to assess the error in a 3D framework, where the cost of computing the boundary fluxes and their equilibration is usually extremely large. Moreover, it is also important to notice that flux-free estimators only require one array additional to the standard data structure already present in any standard finite element code. This array contains information on the elements that surround every given point. This data is easily determined once and for all when topology is known, and in some codes it is already available (viz. Matlab and Cast3m). In particular and in contrast to hybrid-flux techniques there is no need to have structured the information on edges/faces for the evaluation of fluxes. The remainder of this section is devoted to analyze the main properties of the estimates introduced above.

\subsection{Solvability of the local error equation}

The local equation (12) is solved in each star $\omega^{i}$ in order to compute the local estimate $\tilde{\boldsymbol{e}}^{\omega^{i}}$. Note that the r.h.s. term of (12), $R^{P}\left(\phi^{i}\left(\boldsymbol{v}-\boldsymbol{\pi}^{H} \boldsymbol{v}\right)\right)$, does not coincide with the obvious decomposition of the residue given in equation (11), namely

$$
a_{\omega^{i}}\left(\tilde{\boldsymbol{e}}^{\omega^{i}}, \boldsymbol{v}\right)=R^{P}\left(\phi^{i} \boldsymbol{v}\right) \quad \forall \boldsymbol{v} \in \mathcal{V}_{\omega^{i}}^{h} .
$$

The term $R^{P}\left(\phi^{i} \boldsymbol{v}\right)$ has been replaced in (12) by $R^{P}\left(\phi^{i}\left(\boldsymbol{v}-\pi^{H} \boldsymbol{v}\right)\right)$. This is done to ensure the solvability of the local equation.

Theorem 4 The local problem for the estimate $\tilde{\boldsymbol{e}}^{\omega^{i}}$,

$$
a_{\omega^{i}}\left(\tilde{\boldsymbol{e}}^{\omega^{i}}, \boldsymbol{v}\right)=R^{P}\left(\phi^{i}\left(\boldsymbol{v}-\pi^{H} \boldsymbol{v}\right)\right) \quad \forall \boldsymbol{v} \in \mathcal{V}_{\omega^{i}}^{h},
$$

is solvable.

This Theorem is based on the following one, which can be found in $[18$, Thm 9.2.30],

Theorem 5 Let $\mathcal{V}$ be a Hilbert space and $a(\cdot, \cdot)$ be a bilinear form acting on $\mathcal{V} \times \mathcal{V}$. Let also $\mathcal{V}=$ ker $a \oplus \hat{\mathcal{V}}$ be a decomposition of $\mathcal{V}$, that is, for any given $\boldsymbol{v} \in \mathcal{V}$, there exists a unique pair $\left(\boldsymbol{v}_{a}, \hat{\boldsymbol{v}}\right) \in \operatorname{ker} a \times \widehat{\mathcal{V}}$ such that $\boldsymbol{v}=\boldsymbol{v}_{a}+\hat{\boldsymbol{v}}$, where

$$
\text { ker } a:=\left\{\boldsymbol{v}_{a} \in \mathcal{V} \mid a\left(\boldsymbol{v}_{a}, \boldsymbol{w}\right)=0 \forall \boldsymbol{w} \in \mathcal{V}\right\} .
$$

Assume also that the bilinear form $a(\cdot, \cdot)$ is coercive on $\hat{\mathcal{V}}$, that is

$$
\exists \gamma>0 \text { such that } a(\hat{\boldsymbol{v}}, \hat{\boldsymbol{v}}) \geq \gamma\|\hat{\boldsymbol{v}}\|^{2} \quad \forall \hat{v} \in \hat{\mathcal{V}} .
$$

Then, the variational problem: find $u \in \mathcal{V}$ such that

$$
a(\boldsymbol{u}, \boldsymbol{v})=l(\boldsymbol{v}) \quad \forall \boldsymbol{v} \in \mathcal{V}
$$


is solvable if and only if the following compatibility condition holds:

$$
l(\boldsymbol{v})=0 \quad \forall \boldsymbol{v} \in \operatorname{ker} a .
$$

Solvability of a variational problem depends on the verification of the compatibility condition for the functions in the kernel of the bilinear operator. Thus, solvability of equation (12) depends on the model problem at hand. Here the mechanical problem is discussed but the following remark is concerned with scalar equations.

Remark 6 Consider the scalar diffusion-reaction equation. The bilinear form for this problem is

$$
a(u, v)=\int_{\Omega} \nu \nabla u \cdot \nabla v+\mu u v d \Omega
$$

for a strictly positive real coefficient $\nu \in \mathcal{L}^{\infty}(\Omega)$, an a nonnegative real coefficient $\mu \in \mathcal{L}^{\infty}(\Omega)$, and its restriction to a star $\omega^{i}$ is, as previously, denoted by $a_{\omega^{i}}(u, v)$. A strictly positive reaction term in $a_{\omega^{i}}(u, v)$ ensures the solvability of local equation (12) since the ker $a_{\omega^{i}}=\emptyset$. For $\left.\mu\right|_{\omega^{i}}=0$, the kernel of the bilinear operator $a_{\omega^{i}}(\cdot, \cdot)$ is the one dimensional space of constants, $\mathbb{P}^{0}\left(\omega^{i}\right)$. Then, equation (12) is solvable if and only if the compatibility condition holds, namely

$$
R^{P}\left(\phi^{i} c\right)=c R^{P}\left(\phi^{i}\right)=0 \quad \forall c \in \mathbb{P}^{0}\left(\omega^{i}\right),
$$

which follows from the orthogonality of the primal residual to the finite element space $\mathcal{V}^{H}$, since $\phi^{i} \in \mathcal{V}^{H}$.

The bilinear form for the elasticity problem is defined in (3) and the kernel of its restriction to $\omega^{i}, a_{\omega^{i}}(\cdot, \cdot)$, is defined by the solid rigid motions, that is, the zero energy modes. In $1 \mathrm{D}$, the rigid body motions are only translations, that is, the one dimensional space of constants, $\mathbb{P}^{0}\left(\omega^{i}\right)$. In this case, as in the scalar (thermal) problem, for $c \in \mathbb{P}^{0}\left(\omega^{i}\right), R^{P}\left(\phi^{i} c\right)=c R^{P}\left(\phi^{i}\right)=0$ due to the Galerkin orthogonality and therefore the compatibility equation holds and equation (12) is solvable.

However for 2D and 3D mechanical problems, the solid rigid motions include also rotations. For instance in a 2D setup, the kernel of $a_{\omega^{i}}(\cdot, \cdot)$ is a space of three dimensions generated by two translations $\boldsymbol{t}_{x}$ and $\boldsymbol{t}_{y}$ and one rotation $\boldsymbol{\theta}$. The rotation is a linear function and consequently $\phi^{i} \boldsymbol{\theta}$ does not always belong to $\mathcal{V}^{H}$ (for instance, for linear triangular elements, $\phi^{i} \boldsymbol{\theta} \notin \mathcal{V}^{H}$ ), and hence $R^{P}\left(\phi^{i} \boldsymbol{\theta}\right)$ is not necessarily zero. Thus, since the compatibility condition does not hold in general, it can not be guaranteed that equation (12) is solvable. From a mechanical viewpoint, the forces associated with $R^{P}\left(\phi^{i} \boldsymbol{v}\right)$ are not equilibrated (the sum of forces is zero but the sum of moments does not vanish). 
For domains with piecewise linear boundaries, a natural way to circumvent this problem is to consider higher-order Lagrange elements. If $\phi^{i}$ are firstorder Lagrange (linear, bilinear or trilinear) shape functions associated to the set of vertices of the finite mesh, since $\boldsymbol{\theta}$ is also a linear combination of firstorder Lagrange shape functions then $\phi^{i} \boldsymbol{\theta} \in \mathcal{V}^{H}$ and thus the compatibility condition is verified. Thus, equation (12) is solvable.

However, this is no longer valid for first-order Lagrange elements nor for domains with curved boundaries where rotation, $\boldsymbol{\theta}$, is not characterized by a linear combination of first-order shape functions (recall that an isoparametric transformation is used for curved elements). In this case, an alternative is to correct the r.h.s. of (12) to ensure that the compatibility condition is verified also for rotations.

Since $R^{P}(\boldsymbol{v})=R^{P}\left(\boldsymbol{v}-\pi^{H} \boldsymbol{v}\right)$ for all $\boldsymbol{v} \in \mathcal{V}$ by Galerkin orthogonality, the partition defined by (11) can be redefined as

$$
R^{P}(\boldsymbol{v})=\sum_{i=1}^{\mathrm{n}_{\mathrm{np}}} R^{P}\left(\phi^{i}\left(\boldsymbol{v}-\boldsymbol{\pi}^{H} \boldsymbol{v}\right)\right),
$$

which leads to equation (12). In this case, any rigid solid motion $\boldsymbol{v}_{\mathrm{rm}}$ belongs to $\mathcal{V}^{H}$, thus $\boldsymbol{v}_{\mathrm{rm}}-\boldsymbol{\pi}^{H} \boldsymbol{v}_{\mathrm{rm}}=\mathbf{0}$ and consequently problem (12) is solvable.

\subsection{The upper bound property}

The following results summarize the basic property that most residual type error estimators based in a flux equilibration technique verify, see references $[19,20]$, and also proves the upper bound property of this type of estimates.

Lemma 7 Any estimate $\tilde{\boldsymbol{e}} \in \mathcal{V}_{\text {brok }}^{h}$ verifying the weak error equation

$$
a(\tilde{\boldsymbol{e}}, \boldsymbol{v})=R^{P}(\boldsymbol{v}) \quad \forall \boldsymbol{v} \in \mathcal{V}^{h},
$$

is such that the norm of $\tilde{\boldsymbol{e}}$ is an upper bound of the energy norm of the reference error, that is

$$
\|\tilde{\boldsymbol{e}}\|^{2} \geq\left\|\boldsymbol{e}_{h}\right\|^{2}
$$

PROOF. First the following trivial expansion is performed

$$
0 \leq\left\|\boldsymbol{e}_{h}-\tilde{\boldsymbol{e}}\right\|^{2}=\left\|\boldsymbol{e}_{h}\right\|^{2}+\|\tilde{\boldsymbol{e}}\|^{2}-2 a\left(\tilde{\boldsymbol{e}}, \boldsymbol{e}_{h}\right) .
$$

Now, replacing $\boldsymbol{v}$ by $\boldsymbol{e}_{h}$ in (19) and using equality $R^{P}\left(\boldsymbol{e}_{h}\right)=\left\|\boldsymbol{e}_{h}\right\|^{2}$, see (6), the upper bound property is obtained as follows

$$
0 \leq\left\|\boldsymbol{e}_{h}-\tilde{\boldsymbol{e}}\right\|^{2}=\left\|\boldsymbol{e}_{h}\right\|^{2}+\|\tilde{\boldsymbol{e}}\|^{2}-2 R^{P}\left(\boldsymbol{e}_{h}\right)=\|\tilde{\boldsymbol{e}}\|^{2}-\left\|\boldsymbol{e}_{h}\right\|^{2} .
$$


Thus, to prove that $\mathrm{E}_{\mathrm{u}}\left[\boldsymbol{e}_{h}\right]$ is an upper bound of the energy norm of the error, it is only necessary to check that the global estimate $\tilde{\boldsymbol{e}}$, defined in (13), verifies equation (19).

Theorem 8 The estimate $\tilde{\boldsymbol{e}}=\sum_{i=1}^{n_{n p}} \tilde{\boldsymbol{e}}^{\omega^{i}}$, where $\tilde{\boldsymbol{e}}^{\omega^{i}}$ is the solution of the local problem given in (12), is such that

$$
\mathrm{E}_{\mathrm{u}}\left[\boldsymbol{e}_{h}\right]=\|\tilde{\boldsymbol{e}}\|^{2} \geq\left\|\boldsymbol{e}_{h}\right\|^{2}
$$

PROOF. Using equations (12) and (13) together with Galerkin orthogonality

$$
\begin{aligned}
a(\tilde{\boldsymbol{e}}, \boldsymbol{v}) & =\sum_{i=1}^{\mathrm{n}_{\mathrm{np}}} a\left(\tilde{\boldsymbol{e}}^{\omega^{i}}, \boldsymbol{v}\right)=\sum_{i=1}^{\mathrm{n}_{\mathrm{np}}} a_{\omega^{i}}\left(\tilde{\boldsymbol{e}}^{\omega^{i}}, \boldsymbol{v}\right)=\sum_{i=1}^{\mathrm{n}_{\mathrm{np}}} R^{P}\left(\phi^{i}\left(\boldsymbol{v}-\pi^{H} \boldsymbol{v}\right)\right) \\
& =R^{P}\left(\sum_{i=1}^{\mathrm{n}_{\mathrm{np}}} \phi^{i}\left(\boldsymbol{v}-\pi^{H} \boldsymbol{v}\right)\right)=R^{P}(\boldsymbol{v})-R^{P}\left(\boldsymbol{\pi}^{H} \boldsymbol{v}\right)=R^{P}(\boldsymbol{v}) \quad \forall \boldsymbol{v} \in \mathcal{V}^{h}
\end{aligned}
$$

And the proof is concluded using Lemma 7.

\subsection{Lower bound by postprocessing}

The following theorem, see [16] for a detailed proof, shows that every continuous function yields a lower bound of the energy norm of the error. In particular those obtained by post-processing as indicated in Section 3.3. Obviously, for an arbitrary estimate $\tilde{\boldsymbol{e}}_{\text {cont }}$, the corresponding lower bound may have a very poor quality. The best choice for $\tilde{\boldsymbol{e}}_{\text {cont }}$ is either $\boldsymbol{e}$ or $\boldsymbol{e}_{h}$, in order to obtain $\mathrm{E}_{1}$ equal to $\|\boldsymbol{e}\|^{2}$ or $\left\|\boldsymbol{e}_{h}\right\|^{2}$. Therefore, to obtain sharp lower bounds, the estimate $\tilde{\boldsymbol{e}}_{\text {cont }}$ must be a good approximation of the actual error (either exact or reference).

Theorem 9 For any $\tilde{\boldsymbol{e}}_{\text {cont }} \in \mathcal{V}$, a lower bound of the energy norm of the exact error is recovered as

$$
0 \leq \mathrm{E}_{\mathrm{l}}[\boldsymbol{e}]:=\frac{\left(R^{P}\left(\tilde{\boldsymbol{e}}_{\text {cont }}\right)\right)^{2}}{\left\|\tilde{\boldsymbol{e}}_{\text {cont }}\right\|^{2}} \leq\|\boldsymbol{e}\|^{2} .
$$

Moreover, if $\tilde{\boldsymbol{e}}_{\text {cont }} \in \mathcal{V}^{h} \subset \mathcal{V}$, the lower bound is also a lower bound with respect to the energy norm of the reference error, that is,

$$
0 \leq \mathrm{E}_{\mathrm{l}}\left[\boldsymbol{e}_{h}\right]:=\frac{\left(R^{P}\left(\tilde{\boldsymbol{e}}_{\mathrm{cont}}\right)\right)^{2}}{\left\|\tilde{\boldsymbol{e}}_{\mathrm{cont}}\right\|^{2}} \leq\left\|\boldsymbol{e}_{h}\right\|^{2} \leq\|\boldsymbol{e}\|^{2} .
$$




\subsection{Enhancing the lower bound}

The continuous function $\tilde{\boldsymbol{e}}_{\text {cont }}$ is obtained by performing only local computations, consequently the corresponding estimate $\mathrm{E}_{1}$ does not account for pollution errors. The unestimated part of error, $\boldsymbol{e}-\tilde{\boldsymbol{e}}_{\text {cont }}$, includes the pollution effects and it is denoted as global error. In order to assess pollution, the equation for the global error is solved on the coarse mesh following the methodology proposed in [17]. Thus, $\tilde{\boldsymbol{e}}^{\mathrm{G}} \in \mathcal{V}^{H}$ is computed using equation (16) and the enhanced lower bound estimate, $\mathrm{E}_{1}^{\mathrm{G}}\left[\boldsymbol{e}_{h}\right]$, is obtained using (17). The following theorem states that $\mathrm{E}_{1}^{\mathrm{G}}\left[\boldsymbol{e}_{h}\right]$ is also a lower bound of the squared error energy norm.

Theorem 10 Let $\tilde{\boldsymbol{e}}^{\mathrm{G}} \in \mathcal{V}^{H}$ be the solution of

$$
a\left(\tilde{\boldsymbol{e}}^{\mathrm{G}}, \boldsymbol{v}\right)=-a\left(\tilde{\boldsymbol{e}}_{\mathrm{cont}}, \boldsymbol{v}\right) \quad \forall \boldsymbol{v} \in \mathcal{V}^{H}
$$

where $\tilde{\boldsymbol{e}}_{\mathrm{cont}} \in \mathcal{V}^{h}$ is any continuous estimate. Then

$$
\mathrm{E}_{\mathrm{l}}^{\mathrm{G}}\left[\boldsymbol{e}_{h}\right]:=\frac{\left(R^{P}\left(\tilde{\boldsymbol{e}}_{\mathrm{cont}}\right)\right)^{2}}{\left\|\tilde{\boldsymbol{e}}_{\mathrm{cont}}\right\|^{2}-\left\|\tilde{\boldsymbol{e}}^{\mathrm{G}}\right\|^{2}} \leq\left\|\boldsymbol{e}_{h}\right\|^{2} .
$$

PROOF. Let $\tilde{\boldsymbol{e}}_{\mathrm{cont}}^{\mathrm{G}}:=\tilde{\boldsymbol{e}}_{\mathrm{cont}}+\tilde{\boldsymbol{e}}^{\mathrm{G}}$, thus using Theorem 9,

$$
\frac{\left(R^{P}\left(\tilde{\boldsymbol{e}}_{\mathrm{cont}}^{\mathrm{G}}\right)\right)^{2}}{\left\|\tilde{\boldsymbol{e}}_{\mathrm{cont}}^{\mathrm{G}}\right\|^{2}} \leq\left\|\boldsymbol{e}_{h}\right\|^{2} .
$$

First, the residue is modified as

$$
R^{P}\left(\tilde{\boldsymbol{e}}_{\text {cont }}^{\mathrm{G}}\right)=R^{P}\left(\tilde{\boldsymbol{e}}_{\text {cont }}\right)+R^{P}\left(\tilde{\boldsymbol{e}}^{\mathrm{G}}\right)=R^{P}\left(\tilde{\boldsymbol{e}}_{\text {cont }}\right),
$$

because the weak residue vanishes for every function in the finite element space $\mathcal{V}^{H}$ (Galerkin orthogonality). And second, the proof is completed replacing the denominator by

$$
\begin{aligned}
\left\|\tilde{\boldsymbol{e}}_{\mathrm{cont}}^{\mathrm{G}}\right\|^{2} & =\left\|\tilde{\boldsymbol{e}}_{\text {cont }}\right\|^{2}+\left\|\tilde{\boldsymbol{e}}^{\mathrm{G}}\right\|^{2}+2 a\left(\tilde{\boldsymbol{e}}_{\mathrm{cont}}, \tilde{\boldsymbol{e}}^{\mathrm{G}}\right)=\left\|\tilde{\boldsymbol{e}}_{\mathrm{cont}}\right\|^{2}+\left\|\tilde{\boldsymbol{e}}^{\mathrm{G}}\right\|^{2}-2 a\left(\tilde{\boldsymbol{e}}^{\mathrm{G}}, \tilde{\boldsymbol{e}}^{\mathrm{G}}\right) \\
& =\left\|\tilde{\boldsymbol{e}}_{\text {cont }}\right\|^{2}-\left\|\tilde{\boldsymbol{e}}^{\mathrm{G}}\right\|^{2},
\end{aligned}
$$

where equation (16) is used replacing $\boldsymbol{v}$ by $\tilde{\boldsymbol{e}}^{\mathrm{G}}$.

It is worth noting that the non-enhanced lower bound $\mathrm{E}_{\mathrm{l}}\left[\boldsymbol{e}_{h}\right]$ is also an error estimate. The computation of $\tilde{\boldsymbol{e}}^{\mathrm{G}}$ and $\mathrm{E}_{1}^{\mathrm{G}}\left[\boldsymbol{e}_{h}\right]$ is only performed to improve the quality of the error assessment: the value of the enhanced estimate is larger and the lower bound property is conserved. Therefore, the enhanced estimate, $\mathrm{E}_{1}^{\mathrm{G}}\left[\boldsymbol{e}_{h}\right]$, is sharper. 


\section{Comparison with other existing methods}

References [8-10] use apparently similar techniques to obtain upper bounds of the error in the context of a scalar model problem. This section is devoted to compare these techniques with the approach proposed in this paper. The rationale in [8-10] is to decompose the bilinear form $a(\cdot, \cdot)$ in a sum of local contributions associated with each star. That is, weighted local bilinear forms $a^{\mathrm{w}^{i}}(\cdot, \cdot)$ are introduced such that

$$
a(\boldsymbol{u}, \boldsymbol{v})=\sum_{i=1}^{\mathrm{n}_{\mathrm{np}}} \int_{\omega^{i}} \mathrm{w}^{i} \boldsymbol{\sigma}(\boldsymbol{u}): \boldsymbol{\varepsilon}(\boldsymbol{v}) d \Omega=: \sum_{i=1}^{\mathrm{n}_{\mathrm{np}}} a^{\mathrm{w}^{i}}(\boldsymbol{u}, \boldsymbol{v}),
$$

where the weights $\mathrm{w}^{i}$ account for the overlapping of the stars verifying the partition of the unity property:

$$
\sum_{i=1}^{\mathrm{n}_{\mathrm{np}}} \mathrm{w}^{i}=1
$$

The local norm induced by $a^{\mathrm{w}^{i}}(\cdot, \cdot)$ is denoted by $\|\cdot\|_{\mathrm{w}^{i}}$, that is, $\|\boldsymbol{v}\|_{\mathrm{w}^{i}}^{2}:=$ $a^{\mathrm{w}^{i}}(\boldsymbol{v}, \boldsymbol{v})$.

Two different choices for $\mathrm{w}^{i}$ have been considered. In [9], for each element $\Omega_{k}$ of the star $\omega^{i}$, the proposed weight is $\left.\mathrm{w}^{i}\right|_{\Omega_{k}}=\left(1 / \sigma_{k}\right)$ where $\sigma_{k}$ is the number of vertices of the element $\Omega_{k}$. In $[8,10]$, the local weights are the shape functions, $\mathrm{w}^{i}=\phi^{i}$.

Once the bilinear form is decomposed into local contributions, the local estimates $\hat{\boldsymbol{e}}^{\omega^{i}} \in \mathcal{V}_{\omega^{i}}^{h}$ are computed solving the local equation

$$
a^{\mathrm{w}^{i}}\left(\hat{\boldsymbol{e}}^{\omega^{i}}, \boldsymbol{v}\right)=R^{P}\left(\phi^{i}\left(\boldsymbol{v}-\pi^{H} \boldsymbol{v}\right)\right) \quad \forall \boldsymbol{v} \in \mathcal{V}_{\omega^{i}}^{h}
$$

Remark 11 In fact, in [8-10] the r.h.s. of (21) does not include the projection $\pi^{H} \boldsymbol{v}$. This is because these papers are only concerned with scalar (thermal) problems and, consequently, the solvability issues discussed in Section 4.1 are not relevant.

The upper bound of $\left\|\boldsymbol{e}_{h}\right\|^{2}$ is obtained adding the local weighted norms of $\hat{\boldsymbol{e}}^{\omega^{i}}$, that is

$$
\hat{\mathrm{E}}_{\mathrm{u}}\left[\boldsymbol{e}_{h}\right]:=\sum_{i=1}^{\mathrm{n}_{\mathrm{np}}}\left\|\hat{\boldsymbol{e}}^{\omega^{i}}\right\|_{\mathrm{w}^{i}}^{2} \geq\left\|\boldsymbol{e}_{h}\right\|^{2} .
$$

The strategy to obtain the upper bound estimate is summarized in Figure 4.

Note that $\mathrm{E}_{\mathrm{u}}\left[\boldsymbol{e}_{h}\right]$ and $\hat{\mathrm{E}}_{\mathrm{u}}\left[\boldsymbol{e}_{h}\right]$ are computed with completely different expressions. The former is the norm of a sum and the latter is the sum of local norms. 
Step 1.- Compute $\boldsymbol{u}_{H} \in \mathcal{U}^{H}$.

Step 2.- For $i=1, \ldots, \mathrm{n}_{\mathrm{np}}$ compute $\hat{\boldsymbol{e}}^{\omega^{i}} \in \mathcal{V}_{\omega^{i}}^{h}$ such that

$$
a^{\mathrm{w}^{i}}\left(\hat{\boldsymbol{e}}^{\omega^{i}}, \boldsymbol{v}\right)=R^{P}\left(\phi^{i}\left(\boldsymbol{v}-\boldsymbol{\pi}^{H} \boldsymbol{v}\right)\right) \quad \forall \boldsymbol{v} \in \mathcal{V}_{\omega^{i}}^{h}
$$

Step 3.- Compute the upper bound as

$$
\hat{\mathrm{E}}_{\mathrm{u}}\left[\boldsymbol{e}_{h}\right]:=\sum_{i=1}^{\mathrm{n}_{\mathrm{np}}}\left\|\hat{\boldsymbol{e}}^{\omega^{i}}\right\|_{\mathrm{w}^{i}}^{2} \geq\left\|\boldsymbol{e}_{h}\right\|^{2} .
$$

Fig. 4. Alternative upper bound for the squared energy norm of the reference error

The only difference between equations (12) and (21) is the bilinear form in the 1.h.s term. However, the upper bounds $\mathrm{E}_{\mathrm{u}}\left[\boldsymbol{e}_{h}\right]$ and $\hat{\mathrm{E}}_{\mathrm{u}}\left[\boldsymbol{e}_{h}\right]$ have a different expression and, consequently, the analysis of the properties of the estimates follows a different strategy.

The following theorem states that $\hat{\mathrm{E}}_{\mathrm{u}}\left[\boldsymbol{e}_{h}\right]$ is indeed an upper bound of the squared energy norm of the error.

Theorem 12 Let $\hat{\boldsymbol{e}}^{\omega^{i}}$ be the solution of the local equation (21) where $\mathrm{w}^{i}$ are a partition of unity. Then,

$$
\hat{\mathrm{E}}_{\mathrm{u}}\left[\boldsymbol{e}_{h}\right]=\sum_{i=1}^{n_{n p}}\left\|\hat{\boldsymbol{e}}^{\omega^{i}}\right\|_{\mathrm{w}^{i}}^{2}
$$

is an upper bound of the squared energy norm of the reference error, namely, $\hat{\mathrm{E}}_{\mathrm{u}}\left[\boldsymbol{e}_{h}\right] \geq\left\|\boldsymbol{e}_{h}\right\|^{2}$.

PROOF. The decomposition of the bilinear form $a(\cdot, \cdot)$ defined in (20) leads to the following decomposition of the energy norm

$$
\|\boldsymbol{v}\|=a(\boldsymbol{v}, \boldsymbol{v})^{\frac{1}{2}}=\left(\sum_{i=1}^{\mathrm{n}_{\mathrm{np}}} a^{\mathrm{w}^{i}}(\boldsymbol{v}, \boldsymbol{v})\right)^{\frac{1}{2}}=\left(\sum_{i=1}^{\mathrm{n}_{\mathrm{np}}}\|v\|_{\mathrm{w}^{i}}^{2}\right)^{\frac{1}{2}} .
$$

Moreover, using equations (11) and (21) together with Galerkin orthogonality, the squared energy norm of the reference error is rewritten as

$$
\left\|\boldsymbol{e}_{h}\right\|^{2}=a\left(\boldsymbol{e}_{h}, \boldsymbol{e}_{h}\right)=R^{P}\left(\boldsymbol{e}_{h}\right)=\sum_{i=1}^{\mathrm{n}_{\mathrm{np}}} R^{P}\left(\phi^{i}\left(\boldsymbol{e}_{h}-\pi^{H} \boldsymbol{e}_{h}\right)\right)=\sum_{i=1}^{\mathrm{n}_{\mathrm{np}}} a^{\mathrm{w}^{i}}\left(\hat{\boldsymbol{e}}^{\omega^{i}}, \boldsymbol{e}_{h}\right) .
$$

Combining these two decompositions and with repeated use of the Cauchy- 
Schwartz inequality the proof is completed

$$
\begin{aligned}
\left\|\boldsymbol{e}_{h}\right\|^{2} & =\left|\sum_{i=1}^{\mathrm{n}_{\mathrm{np}}} a^{\mathrm{w}^{i}}\left(\hat{\boldsymbol{e}}^{\omega^{i}}, \boldsymbol{e}_{h}\right)\right| \leq \sum_{i=1}^{\mathrm{n}_{\mathrm{np}}}\left|a^{\mathrm{w}^{i}}\left(\hat{\boldsymbol{e}}^{\omega^{i}}, \boldsymbol{e}_{h}\right)\right| \leq \sum_{i=1}^{\mathrm{n}_{\mathrm{np}}}\left\|\hat{\boldsymbol{e}}^{\omega^{i}}\right\|_{\mathrm{w}^{i}}\left\|\boldsymbol{e}_{h}\right\|_{\mathrm{w}^{i}} \\
& \leq\left(\sum_{i=1}^{\mathrm{n}_{\mathrm{np}}}\left\|\hat{\boldsymbol{e}}^{\omega^{i}}\right\|_{\mathrm{w}^{i}}^{2}\right)^{\frac{1}{2}}\left(\sum_{i=1}^{\mathrm{n}_{\mathrm{np}}}\left\|\boldsymbol{e}_{h}\right\|_{\mathrm{w}^{i}}^{2}\right)^{\frac{1}{2}} \leq \hat{\mathrm{E}}_{\mathrm{u}}\left[\boldsymbol{e}_{h}\right]^{\frac{1}{2}}\left\|\boldsymbol{e}_{h}\right\|
\end{aligned}
$$

Remark 13 The repeated use of the Cauchy-Schwartz inequality in the proof of Theorem 12 suggests that the obtained upper bound is not as sharp as the upper bound associated with the estimate $\tilde{\boldsymbol{e}}$. The numerical examples confirm this impression: the estimate $\mathrm{E}_{\mathrm{u}}\left[\boldsymbol{e}_{h}\right]$ is usually sharper than $\hat{\mathrm{E}}_{\mathrm{u}}\left[\boldsymbol{e}_{h}\right]$.

\section{Bounds of the error in outputs of interest}

As shown in Section 2.3, in order to estimate bounds of the error in the output of interest $l^{\mathcal{O}}\left(\boldsymbol{e}_{h}\right)$, upper and lower bounds of $\left\|\boldsymbol{z}_{h}^{ \pm}\right\|$are necessary instead of bounds of $\left\|\boldsymbol{e}_{h}\right\|$. Recall that $\boldsymbol{z}_{h}^{ \pm}=\kappa \boldsymbol{e}_{h} \pm \frac{1}{\kappa} \boldsymbol{\epsilon}_{h}$ where the error of the primal and dual problems are involved. This section presents and discusses the particular evaluation of $\mathrm{E}_{\mathrm{u}}\left[\boldsymbol{z}_{h}^{ \pm}\right]$and $\mathrm{E}_{\mathrm{l}}\left[\boldsymbol{z}_{h}^{ \pm}\right]$. These values, as indicated by equation (10), allow to bound $\left\|\boldsymbol{z}_{h}^{ \pm}\right\|$. Note also that bounds for the output of interest, $l^{\mathcal{O}}\left(\boldsymbol{u}_{h}\right)$, can be computed adding $l^{\mathcal{O}}\left(\boldsymbol{u}_{H}\right)$ to each term of inequality (10).

\subsection{Upper bound computation of $\left\|\boldsymbol{z}_{h}^{ \pm}\right\|$}

In order to determine $\mathrm{E}_{\mathrm{u}}\left[\boldsymbol{z}_{h}^{ \pm}\right]$the error estimate of both the primal and dual problem are necessary. Section 3 describes the evaluation of the primal error estimate. The same methodology is used to estimate the dual error, $\tilde{\boldsymbol{\epsilon}}$, by adding local estimates $\tilde{\boldsymbol{\epsilon}}^{\omega^{i}} \in \mathcal{V}_{\omega^{i}}^{h}$ computed from

$$
a_{\omega^{i}}\left(\boldsymbol{v}, \tilde{\boldsymbol{\epsilon}}^{\omega^{i}}\right)=R^{D}\left(\phi^{i}\left(\boldsymbol{v}-\pi^{H} \boldsymbol{v}\right)\right) \quad \forall \boldsymbol{v} \in \mathcal{V}_{\omega^{i}}^{h}
$$

Then, the upper bound for $\left\|\boldsymbol{z}_{h}^{ \pm}\right\|^{2}, \mathrm{E}_{\mathrm{u}}\left[\boldsymbol{z}_{h}^{ \pm}\right]$, is obtained as summarized in Figure 5 and based on the following Lemma.

Lemma 14 The estimate $\mathrm{E}_{\mathrm{u}}\left[\boldsymbol{z}_{h}^{ \pm}\right]:=2\|\tilde{\boldsymbol{e}}\|\|\tilde{\boldsymbol{\epsilon}}\| \pm 2 a(\tilde{\boldsymbol{e}}, \tilde{\boldsymbol{\epsilon}})$ is such that

$$
\mathrm{E}_{\mathrm{u}}\left[\boldsymbol{z}_{h}^{ \pm}\right] \geq\left\|\boldsymbol{z}_{h}^{ \pm}\right\|^{2} .
$$


Step 1.- For $i=1, \ldots, \mathrm{n}_{\mathrm{np}}$, compute the local estimates $\tilde{\boldsymbol{e}}^{\omega^{i}}, \tilde{\boldsymbol{\epsilon}}^{\omega^{i}} \in \mathcal{V}_{\omega^{i}}^{h}$ such that

$$
\begin{array}{ll}
a_{\omega^{i}}\left(\tilde{\boldsymbol{e}}^{\omega^{i}}, \boldsymbol{v}\right)=R^{P}\left(\phi^{i}\left(\boldsymbol{v}-\boldsymbol{\pi}^{H} \boldsymbol{v}\right)\right) & \forall \boldsymbol{v} \in \mathcal{V}_{\omega^{i}}^{h}, \\
a_{\omega^{i}}\left(\boldsymbol{v}, \tilde{\boldsymbol{\epsilon}}^{\omega^{i}}\right)=R^{D}\left(\phi^{i}\left(\boldsymbol{v}-\boldsymbol{\pi}^{H} \boldsymbol{v}\right)\right) & \forall \boldsymbol{v} \in \mathcal{V}_{\omega^{i}}^{h}
\end{array}
$$

Step 2.- Define the global estimates $\tilde{\boldsymbol{e}}$ and $\tilde{\boldsymbol{\epsilon}}$ as

$$
\tilde{\boldsymbol{e}}=\sum_{i=1}^{\mathrm{n}_{\mathrm{np}}} \tilde{\boldsymbol{e}}^{\omega^{i}}, \quad \tilde{\boldsymbol{\epsilon}}=\sum_{i=1}^{\mathrm{n}_{\mathrm{np}}} \tilde{\boldsymbol{\epsilon}}^{\omega^{i}} .
$$

Step 3.- Compute the upper bounds of the energy norm of $\boldsymbol{z}_{h}^{ \pm}$as

$$
\mathrm{E}_{\mathrm{u}}\left[\boldsymbol{z}_{h}^{ \pm}\right]=2\|\tilde{\boldsymbol{e}}\|\|\tilde{\boldsymbol{\epsilon}}\| \pm 2 a(\tilde{\boldsymbol{e}}, \tilde{\boldsymbol{\epsilon}}) \geq\left\|\boldsymbol{z}_{h}^{ \pm}\right\|^{2}
$$

Fig. 5. Upper bounds for the squared energy norm of $\boldsymbol{z}_{h}^{ \pm}$

PROOF. Since $a(\cdot, \cdot)$ is a symmetric bilinear form, the following equation for $\boldsymbol{z}_{h}^{ \pm}$holds,

$$
a\left(\boldsymbol{z}_{h}^{ \pm}, \boldsymbol{v}\right)=\kappa R^{P}(\boldsymbol{v}) \pm \frac{1}{\kappa} R^{D}(\boldsymbol{v})=: R^{ \pm}(\boldsymbol{v}) \quad \forall \boldsymbol{v} \in \mathcal{V}^{h}
$$

Then, according to Lemma 7 , an estimate $\tilde{\boldsymbol{z}}^{ \pm} \in \mathcal{V}_{\text {brok }}^{h}$ yields an upper bound of the energy norm of $\boldsymbol{z}_{h}^{ \pm}$if

$$
a\left(\tilde{\boldsymbol{z}}^{ \pm}, \boldsymbol{v}\right)=R^{ \pm}(\boldsymbol{v}) \quad \forall \boldsymbol{v} \in \mathcal{V}^{h}
$$

Recall now that the primal estimate $\tilde{\boldsymbol{e}}$ verifies equation (19), see Lemma 7. Similarly, $\tilde{\boldsymbol{\epsilon}}$ verifies

$$
a(\tilde{\boldsymbol{\epsilon}}, \boldsymbol{v})=R^{D}(\boldsymbol{v}) \quad \forall \boldsymbol{v} \in \mathcal{V}^{h} .
$$

Thus, introducing $\tilde{\boldsymbol{z}}^{ \pm}:=\kappa \tilde{\boldsymbol{e}} \pm \tilde{\boldsymbol{\epsilon}} / \kappa \in \mathcal{V}_{\text {brok }}^{h}$ equation (23) holds true. The proof is completed taking $\kappa^{2}=\|\tilde{\boldsymbol{\epsilon}}\| /\|\tilde{\boldsymbol{e}}\|$ in

$$
\mathrm{E}_{\mathrm{u}}\left[\boldsymbol{z}_{h}^{ \pm}\right]=\left\|\tilde{\boldsymbol{z}}^{ \pm}\right\|^{2}=\kappa^{2}\|\tilde{\boldsymbol{e}}\|^{2}+\frac{1}{\kappa^{2}}\|\tilde{\boldsymbol{\epsilon}}\|^{2} \pm 2 a(\tilde{\boldsymbol{e}}, \tilde{\boldsymbol{\epsilon}})=2\|\tilde{\boldsymbol{e}}\|\|\tilde{\boldsymbol{\epsilon}}\| \pm 2 a(\tilde{\boldsymbol{e}}, \tilde{\boldsymbol{\epsilon}})
$$

\subsection{Lower bound computation of $\left\|\boldsymbol{z}_{h}^{ \pm}\right\|$}

Upper bound estimates of $\tilde{\boldsymbol{z}}^{ \pm}$are also postprocessed to obtain $\tilde{\boldsymbol{z}}_{\text {cont }}^{ \pm}$as described in Section 3.3. The strategy used to obtain lower bounds of $\boldsymbol{z}_{h}^{ \pm}$is summarized in Figure 6. 
Step 1.- Compute $\tilde{\boldsymbol{e}}_{\text {cont }}$ and $\tilde{\boldsymbol{\epsilon}}_{\text {cont }}$ as

$$
\tilde{\boldsymbol{e}}_{\mathrm{cont}}=\pi^{h}\left(\sum_{i=1}^{\mathrm{n}_{\mathrm{np}}} \phi^{i} \tilde{\boldsymbol{e}}^{\omega^{i}}\right) \quad \text { and } \quad \tilde{\boldsymbol{\epsilon}}_{\mathrm{cont}}=\pi^{h}\left(\sum_{i=1}^{\mathrm{n}_{\mathrm{np}}} \phi^{i} \tilde{\boldsymbol{\epsilon}}^{\omega^{i}}\right),
$$

or use a simple average as proposed in [16].

Step 2.- Compute the global enhancements $\tilde{\boldsymbol{e}}^{\mathrm{G}}$ and $\tilde{\boldsymbol{\epsilon}}^{\mathrm{G}}$ in $\mathcal{V}^{H}$ such that

$$
\begin{array}{ll}
a\left(\tilde{\boldsymbol{e}}^{\mathrm{G}}, \boldsymbol{v}\right)=-a\left(\tilde{\boldsymbol{e}}_{\text {cont }}, \boldsymbol{v}\right) & \forall \boldsymbol{v} \in \mathcal{V}^{H}, \\
a\left(\boldsymbol{v}, \tilde{\boldsymbol{\epsilon}}^{\mathrm{G}}\right)=-a\left(\boldsymbol{v}, \tilde{\boldsymbol{\epsilon}}_{\text {cont }}\right) & \forall \boldsymbol{v} \in \mathcal{V}^{H}
\end{array}
$$

Step 3.- Define $\tilde{\boldsymbol{z}}_{\text {cont }}^{ \pm}=\kappa\left(\tilde{\boldsymbol{e}}_{\text {cont }}+\tilde{\boldsymbol{e}}^{\mathrm{G}}\right) \pm \frac{1}{\kappa}\left(\tilde{\boldsymbol{\epsilon}}_{\text {cont }}+\tilde{\boldsymbol{\epsilon}}^{\mathrm{G}}\right)$, and compute the lower bound as

$$
\mathrm{E}_{\mathrm{l}}^{\mathrm{G}}\left[\boldsymbol{z}_{h}^{ \pm}\right]=\frac{\left(R^{ \pm}\left(\tilde{\boldsymbol{z}}_{\text {cont }}^{ \pm}\right)\right)^{2}}{\left\|\tilde{\boldsymbol{z}}_{\text {cont }}^{ \pm}\right\|^{2}} \leq\left\|\boldsymbol{z}_{h}^{ \pm}\right\|^{2}
$$

Fig. 6. Lower bounds for the squared energy norm of $\boldsymbol{z}_{h}^{ \pm}$

Theorem 15 thm:lower-bound-z Let $\tilde{\boldsymbol{e}}^{\mathrm{G}} \in \mathcal{V}^{H}$ and $\tilde{\boldsymbol{\epsilon}}^{\mathrm{G}} \in \mathcal{V}^{H}$ be the global enhancements computed from $\tilde{\boldsymbol{e}}_{\text {cont }} \in \mathcal{V}^{h}$ and $\tilde{\boldsymbol{\epsilon}}_{\text {cont }} \in \mathcal{V}^{h}$, respectively. Then, the estimate $\tilde{\boldsymbol{z}}_{\text {cont }}^{ \pm}:=\kappa\left(\tilde{\boldsymbol{e}}_{\text {cont }}+\tilde{\boldsymbol{e}}^{\mathrm{G}}\right) \pm\left(\tilde{\boldsymbol{\epsilon}}_{\mathrm{cont}}+\tilde{\boldsymbol{\epsilon}}^{\mathrm{G}}\right) / \kappa$ provides a lower bound of the energy norm of $\boldsymbol{z}_{h}^{ \pm}$, that is,

$$
\mathrm{E}_{\mathrm{l}}^{\mathrm{G}}\left[\boldsymbol{z}_{h}^{ \pm}\right]:=\frac{\left(R^{ \pm}\left(\tilde{\boldsymbol{z}}_{\text {cont }}^{ \pm}\right)\right)^{2}}{\left\|\tilde{\boldsymbol{z}}_{\text {cont }}^{ \pm}\right\|^{2}} \leq\left\|\boldsymbol{z}_{h}^{ \pm}\right\|^{2} .
$$

PROOF. The proof is a direct consequence of Theorem 9 if both $\|\tilde{\boldsymbol{e}}\|$ and $\|\tilde{\boldsymbol{\epsilon}}\|$ are non-zero.

The case $\|\tilde{\boldsymbol{e}}\|=0$ or $\|\tilde{\boldsymbol{\epsilon}}\|=0$ is trivial because it implies that either $\boldsymbol{e}=\mathbf{0}$ or $\boldsymbol{\varepsilon}=\mathbf{0}$, and therefore $l^{\mathcal{O}}(\boldsymbol{e})=0$. In this case, the obvious lower bound $\mathrm{E}_{\mathrm{l}}^{\mathrm{G}}\left[\boldsymbol{z}_{h}^{ \pm}\right]$ is 0 .

\section{Computational aspects}

\subsection{Simplified computation of the weak residual}

The simplification proposed in this section defines, in fact, the actual strategy proposed in this paper. As shown next (and as can be observed in the 
examples) there is no loss of certainty. Moreover, the advantages in the computational effort are considerable. Equation (12) is in fact the fundamental equation that is solved repeatedly. The weak residual in its r.h.s. is not trivial to compute because for $\boldsymbol{v} \in \mathcal{V}^{h}$, in general, $\phi^{i} \boldsymbol{v}$ does not belong to the finite element reference space, $\mathcal{V}^{h}$. Note that this is also the case for $\phi^{i}\left(\boldsymbol{v}-\pi^{H} \boldsymbol{v}\right)$. However, the evaluation of the weak residual is drastically simplified if its argument is projected into $\mathcal{V}^{h}$.

For every $\boldsymbol{v} \in \mathcal{V}^{h}$ the following equality holds

$$
R^{P}(\boldsymbol{v})=R^{P}\left(\boldsymbol{\pi}^{h} \boldsymbol{v}\right)=R^{P}\left(\pi^{h}\left(\sum_{i=1}^{\mathrm{n}_{\mathrm{np}}} \phi^{i} \boldsymbol{v}\right)\right)=\sum_{i=1}^{\mathrm{n}_{\mathrm{np}}} R^{P}\left(\pi^{h}\left(\phi^{i} v\right)\right)
$$

Thus the same partition proposed in (11) can be performed with the residual acting on the projection and consequently, equation (12) can be rewritten as find $\tilde{\boldsymbol{e}}^{\omega^{i}} \in \mathcal{V}_{\omega^{i}}^{h}$ such that

$$
a_{\omega^{i}}\left(\tilde{\boldsymbol{e}}^{\omega^{i}}, \boldsymbol{v}\right)=R^{P}\left(\pi^{h}\left(\phi^{i}\left(\boldsymbol{v}-\pi^{H} \boldsymbol{v}\right)\right)\right) \quad \forall \boldsymbol{v} \in \mathcal{V}_{\omega^{i}}^{h}
$$

The behavior of the estimates obtained either introducing the projection, $\pi^{h}$, or not is similar as shown in the numerical examples. However the implementation of the r.h.s. term described in the previous equation is much simpler. This is because the argument of $R^{P}(\cdot)$ is reinjected in the reference space, which is a standard finite element space. Moreover, $\phi^{i} \in \mathcal{V}^{h}$ and $\boldsymbol{v} \in \mathcal{V}^{h}$, thus $\pi^{h}\left(\phi^{i} \boldsymbol{v}\right)$ is computed by a simple product of nodal values of $\phi^{i}$ and $\boldsymbol{v}$ (or $\boldsymbol{v}-\pi^{H} \boldsymbol{v}$ when necessary).

\subsection{Spatial distribution of upper bound estimates}

The upper bound estimate $\mathrm{E}_{\mathrm{u}}\left[\boldsymbol{e}_{h}\right]$ presented in Section 3.2 can be decomposed into positive contributions of each element of the mesh, thus providing local indicators of the value of the local energy norm of the reference error $\left\|\boldsymbol{e}_{h}\right\|_{k}$, that is,

$$
\mathrm{E}_{\mathrm{u}}\left[\boldsymbol{e}_{h}\right]=\|\tilde{\boldsymbol{e}}\|^{2}=\sum_{k=1}^{\mathrm{n}_{\mathrm{e} 1}}\|\tilde{\boldsymbol{e}}\|_{k}^{2},
$$

and $\|\tilde{\boldsymbol{e}}\|_{k}$ is the local indicator for $\left\|\boldsymbol{e}_{h}\right\|_{k}$. 


\section{Numerical examples}

In this section, the behavior of the estimates presented above is analyzed both for thermal and mechanical model problems. Some of the selected examples have been used by other authors to assess performance of similar techniques $[1,3,21]$. The quality of the error estimates is measured with the index

$$
\rho:=\frac{\text { estimated error norm }}{\text { "true" error norm }}-1
$$

where the "true" error is either the exact error (if available) or the reference error. Index $\rho$ is the usual effectivity index minus one. The accuracy of the error estimate is given by the absolute value of $\rho$ and the sign indicates if the estimate is an overestimation (positive $\rho$ ) or an underestimation (negative $\rho$ ) of the true error. For instance, $\rho=2 \%$ indicates that estimated error is larger than the "true" error with a factor 1.02 and $\rho=-3 \%$ means that the "true" error is underestimated by a factor 0.97 .

In the remainder of the section $\rho$ is used to assess the quality of the different estimates, for instance of $\mathrm{E}_{\mathrm{u}}\left[\boldsymbol{e}_{h}\right]$. Note however that $\mathrm{E}_{\mathrm{u}}\left[\boldsymbol{e}_{h}\right]$ is an estimate of the squared energy norm, but the corresponding $\rho$ index is computed using directly the approximation of the error norm (not squared). Moreover, when the exact error, $\boldsymbol{e}$, is known it is always used to compute $\rho$. Thus, $\rho\left(\mathrm{E}_{\mathrm{u}}\left[\boldsymbol{e}_{h}\right]\right)$ is defined either as

$$
\rho\left(\mathrm{E}_{\mathrm{u}}\left[\boldsymbol{e}_{h}\right]\right):=\frac{\sqrt{\mathrm{E}_{\mathrm{u}}\left[\boldsymbol{e}_{h}\right]}}{\|\boldsymbol{e}\|}-1 \quad \text { or as } \quad \rho\left(\mathrm{E}_{\mathrm{u}}\left[\boldsymbol{e}_{h}\right]\right):=\frac{\sqrt{\mathrm{E}_{\mathrm{u}}\left[\boldsymbol{e}_{h}\right]}}{\left\|\boldsymbol{e}_{h}\right\|}-1
$$

depending on the availability of $\boldsymbol{e}$. This definition is extended to the other studied error estimates, for instance $\rho\left(\hat{\mathrm{E}}_{\mathrm{u}}\left[\boldsymbol{e}_{h}\right]\right)$.

\subsection{Thermal problem with energy norm assessment}

First, the scalar benchmark is solved, see $[2,3,16]$. A squared domain, $\Omega=$ ] $0,1[\times] 0,1[$, with Dirichlet homogeneous boundary conditions on $\partial \Omega$ and a source term are chosen such that the exact solution, given in Figure 7, has the following analytical expression

$$
\boldsymbol{u}(x, y)=x^{2}(1-x)^{2}\left(e^{10 x^{2}}-1\right) y^{2}(1-y)^{2}\left(e^{10 y^{2}}-1\right) / 2000 .
$$

The behavior of the energy norm estimates is analyzed comparing the estimates with the exact energy error norm $\|\boldsymbol{e}\|$. Two different non-structured and 

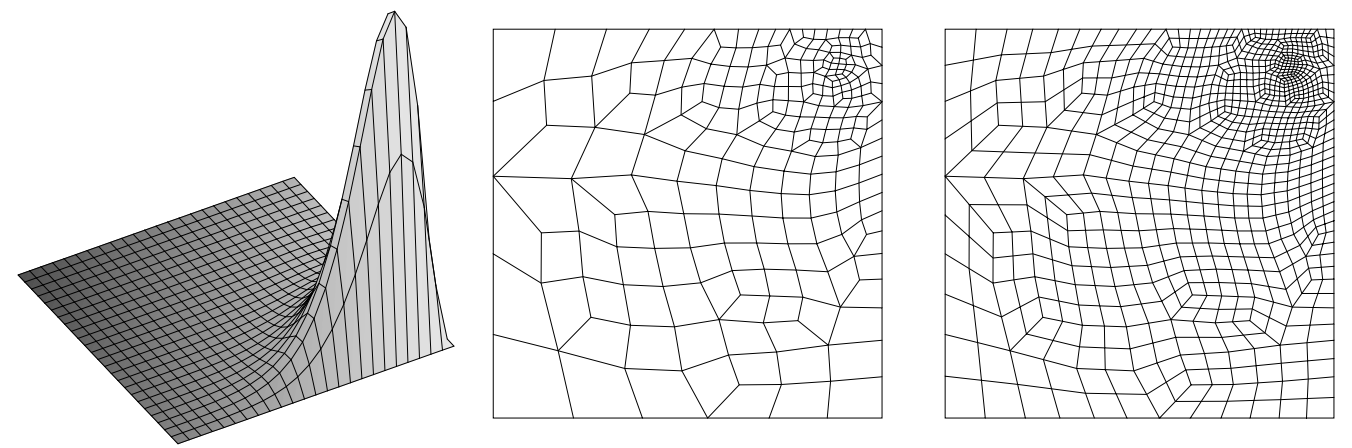

Fig. 7. Thermal problem: exact solution (left) and meshes with 240 dof (center) and 913 dof (right)

non-uniform meshes have been considered, see Figure 7. In both cases, the approximate solution $\boldsymbol{u}_{H}$ is computed using quadrilateral meshes with bilinear interpolation $(p=1)$, and the reference space is associated with a mesh of size $h=H / 4$ (i.e. each element of the $H$-mesh is divided into 16 new elements).

Table 1 presents the $\rho$ indices for estimates of upper bounds. Two versions of $\mathrm{E}_{\mathrm{u}}\left[\boldsymbol{e}_{h}\right]$ are shown, one using the projection $\pi^{h}$ in the r.h.s. term of the local equation (12), as described in Section 7.1 to simplify computations, and another without the proposed projection. Two versions of $\rho\left(\hat{\mathrm{E}}_{\mathrm{u}}\left[\boldsymbol{e}_{h}\right]\right)$ are also evaluated. They correspond to the different weighting functions $\mathrm{w}^{i}$ for the bilinear form in the l.h.s. of equation (21) described in Section 5, and formerly proposed in $[9]\left(\mathrm{w}^{i}=1 / \sigma\right)$ and in $[8,10]\left(\mathrm{w}^{i}=\phi^{i}\right)$.

Paradoxically, in this example, the two upper bounds estimates proposed in this paper (and associated with $\mathrm{E}_{\mathrm{u}}\left[\boldsymbol{e}_{h}\right]$ ) provide negative values of $\rho$. This is because all the presented estimates are upper bounds with respect to the reference error, $\boldsymbol{e}_{h}$, that is, they are larger than the reference error but not necessarily larger than the exact error $\boldsymbol{e}$ which is used to compute $\rho$. Then, even if $\left\|\boldsymbol{e}_{h}\right\|^{2} \leq \mathrm{E}_{\mathrm{u}}\left[\boldsymbol{e}_{h}\right]$ stands, in this case the estimates are very sharp and we have $\left\|\boldsymbol{e}_{h}\right\|^{2}<\mathrm{E}_{\mathrm{u}}\left[\boldsymbol{e}_{h}\right]<\|\boldsymbol{e}\|^{2}$, see Table 1 . The estimates corresponding to $\hat{\mathrm{E}}_{\mathrm{u}}\left[\boldsymbol{e}_{h}\right]$ are far from being sharp, they yield an overestimation of more than $60 \%\left(\right.$ for $\left.\mathrm{w}^{i}=1 / \sigma\right)$ and $20 \%\left(\right.$ for $\left.\mathrm{w}^{i}=\phi^{i}\right)$.

As expected, the effectivity indices of $\mathrm{E}_{\mathrm{u}}\left[\boldsymbol{e}_{h}\right]$ are better than the effectivity indices of $\hat{\mathrm{E}}_{\mathrm{u}}\left[\boldsymbol{e}_{h}\right]$ (for both versions $\mathrm{w}^{i}=1 / \sigma$ and $\mathrm{w}^{i}=\phi^{i}$ ). Table 1 also shows that the proposed projection $\pi^{h}$ in the r.h.s. term of local equation (12), as described in Section 7.1, does not modify substantially the values of effectivity indices. Recall that the use of $\pi^{h}$ simplifies considerably the implementation of the estimator and it is therefore strongly recommended.

Effectivity indices for lower bound estimates are displayed in Tables 2 and 3. Estimates $\hat{\mathrm{E}}_{\mathrm{l}}\left[\boldsymbol{e}_{h}\right]$ and $\hat{\mathrm{E}}_{\mathrm{l}}^{\mathrm{G}}\left[\boldsymbol{e}_{h}\right]$ are computed in the same fashion as $\mathrm{E}_{\mathrm{l}}\left[\boldsymbol{e}_{h}\right]$ but using the continuous function resulting of smoothing $\hat{\boldsymbol{e}}=\sum_{i} \hat{\boldsymbol{e}}^{\omega^{i}}$ instead of 
Table 1

Thermal problem: $\rho$ indices for upper bound energy norm estimates

\begin{tabular}{|c|cc|cccc|}
\hline d.o.f. & $\frac{\|\boldsymbol{e}\|}{\|\boldsymbol{u}\|}$ & $\frac{\left\|\boldsymbol{e}_{h}\right\|}{\|\boldsymbol{e}\|}$ & $\begin{array}{c}\rho\left(\mathrm{E}_{\mathrm{u}}\left[\boldsymbol{e}_{h}\right]\right) \\
\text { without } \boldsymbol{\pi}^{h}\end{array}$ & $\begin{array}{c}\rho\left(\mathrm{E}_{\mathrm{u}}\left[\boldsymbol{e}_{h}\right]\right) \\
\text { with } \boldsymbol{\pi}^{h}\end{array}$ & $\begin{array}{c}\rho\left(\hat{\mathrm{E}}_{\mathrm{u}}\left[\boldsymbol{e}_{h}\right]\right) \\
\mathrm{w}^{i}=1 / \sigma\end{array}$ & $\begin{array}{c}\rho\left(\hat{\mathrm{E}}_{\mathrm{u}}\left[\boldsymbol{e}_{h}\right]\right) \\
\mathrm{w}^{i}=\phi^{i}\end{array}$ \\
\hline 240 & $24.9 \%$ & $95.9 \%$ & $-3.26 \%$ & $-3.34 \%$ & $63.9 \%$ & $23.4 \%$ \\
913 & $15.1 \%$ & $96.6 \%$ & $-2.59 \%$ & $-2.65 \%$ & $67.8 \%$ & $25.8 \%$ \\
\hline
\end{tabular}

Table 2

Thermal problem: $\rho$ indices for lower bound energy norm estimates

\begin{tabular}{|c|cccc|}
\hline d.o.f. & $\rho\left(\mathrm{E}_{\mathrm{l}}\left[\boldsymbol{e}_{h}\right]\right)$ & $\rho\left(\mathrm{E}_{\mathrm{l}}\left[\boldsymbol{e}_{h}\right]\right)$ & $\rho\left(\hat{\mathrm{E}}_{\mathrm{l}}\left[\boldsymbol{e}_{h}\right]\right)$ & $\rho\left(\hat{\mathrm{E}}_{[}\left[\boldsymbol{e}_{h}\right]\right)$ \\
& without $\boldsymbol{\pi}^{h}$ & with $\boldsymbol{\pi}^{h}$ & $\mathrm{w}^{i}=1 / \sigma$ & $\mathrm{w}^{i}=\phi^{i}$ \\
\hline 240 & $-19.9 \%$ & $-19.6 \%$ & $-19.9 \%$ & $-31.0 \%$ \\
913 & $-18.9 \%$ & $-18.6 \%$ & $-18.9 \%$ & $-29.6 \%$ \\
\hline
\end{tabular}

Table 3

Thermal problem: $\rho$ indices for lower bound energy norm estimates with global enhancement

\begin{tabular}{|c|cccc|}
\hline d.o.f. & $\begin{array}{c}\rho\left(\mathrm{E}_{1}^{\mathrm{G}}\left[\boldsymbol{e}_{h}\right]\right) \\
\text { without } \boldsymbol{\pi}^{h}\end{array}$ & $\begin{array}{c}\rho\left(\mathrm{E}_{\mathrm{l}}^{\mathrm{G}}\left[\boldsymbol{e}_{h}\right]\right) \\
\text { with } \boldsymbol{\pi}^{h}\end{array}$ & $\begin{array}{c}\rho\left(\hat{\mathrm{E}}_{\mathrm{l}}^{\mathrm{G}}\left[\boldsymbol{e}_{h}\right]\right) \\
\mathrm{w}^{i}=1 / \sigma\end{array}$ & $\begin{array}{c}\rho\left(\hat{\mathrm{E}}_{1}^{\mathrm{G}}\left[\boldsymbol{e}_{h}\right]\right) \\
\mathrm{w}^{i}=\phi^{i}\end{array}$ \\
\hline 240 & $-6.58 \%$ & $-7.30 \%$ & $-6.58 \%$ & $-4.67 \%$ \\
913 & $-5.79 \%$ & $-6.50 \%$ & $-5.79 \%$ & $-3.97 \%$ \\
\hline
\end{tabular}

$\tilde{\boldsymbol{e}}$, see equation (13). Table 3 shows the results obtained applying the global enhancement discussed in Section 4.4. These results indicate that lower bounds are not sensitive to the original (discontinuous) estimate, which provides the upper bound. All estimates in Table 2 perform similarly. The effect of the global enhancement is however very important: the effectivity indices improve drastically from Table 2 to Table 3 . Recall that in all these tables $\rho$ is computed with respect to the exact error because the exact solution is known.

From a qualitative viewpoint, it is worth noting that the estimated error distribution is in good agreement with the exact error distribution, both for the estimate proposed here $\left(\mathrm{E}_{\mathrm{u}}\left[\boldsymbol{e}_{h}\right]\right)$ and for the estimates proposed in [8-10] (the two versions of $\left.\hat{\mathrm{E}}_{\mathrm{u}}\left[\boldsymbol{e}_{h}\right]\right)$.

Figure 8 shows the spatial distribution of the local effectivity index and the histogram representing the occurrences of local effectivity indices. The histogram shows the number of elements with local efectivity in a given range. The histograms show a good behavior of the estimate if they display a narrow distribution (all elements have similar local effectivity indices) concentrated around $100 \%$. Observe that the local values associated with the estimate $\mathrm{E}_{\mathrm{u}}\left[\boldsymbol{e}_{h}\right]$ proposed here are much more accurate than the values corresponding to $\hat{\mathrm{E}}_{\mathrm{u}}\left[\boldsymbol{e}_{h}\right]$. 

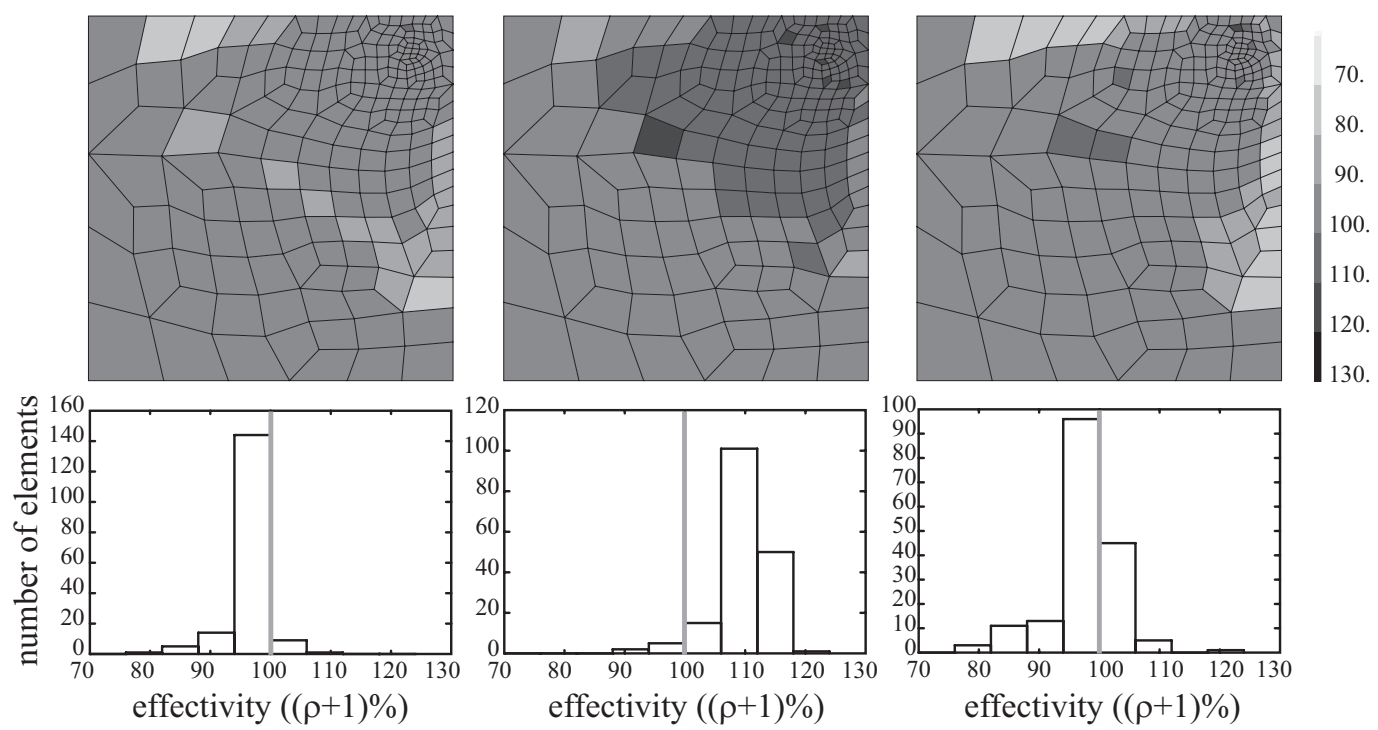

Fig. 8. Thermal problem: spatial distribution of the local effectivity (top) and histograms for local effectivity (bottom). The results correspond to $\mathrm{E}_{\mathrm{u}}\left[\boldsymbol{e}_{h}\right]$ (left), $\hat{\mathrm{E}}_{\mathrm{u}}\left[\boldsymbol{e}_{h}\right]$ with $\mathrm{w}^{i}=\phi^{i}$ (center) and $\mathrm{E}_{\mathrm{l}}^{\mathrm{G}}\left[\boldsymbol{e}_{h}\right]$ (right)

Remark 16 Elements with a small local error are not taken into account. Because the areas where error is small are not interesting from an adaptive viewpoint. Moreover, in these areas, small defaults in the error assessment lead to very bad effectivity indices (small absolute error but large relative error). Here, the criterion used is to suppress in the histograms elements such that the local error norm is lower than $\left\|\boldsymbol{e}_{h}\right\| / 4 n_{e l}$ (being $n_{e l}$ the number of elements). That results on neglecting $20 \%$ of the elements approximately.

\subsection{Thin plate energy error assessment}

A square thin plate with two holes proposed in [22] is considered next. This is a plane-stress linear elastic problem loaded with a horizontal unit tension along the vertical edges $\Gamma_{0}$, see Figure 9 . Note that the solution of this problem has corner singularities due to the interior rectangular cut-outs. Due to symmetry, only one fourth of the domain is analyzed. Values for Young's modulus and Poisson ratio are set to 1 and 0.3 , respectively.

Two meshes are considered, a coarse uniform mesh with 70 nodes and a finer one with 850 nodes, adapted heuristically. Error estimates $\mathrm{E}_{\mathrm{u}}\left[\boldsymbol{e}_{h}\right]$ and $\mathrm{E}_{1}^{\mathrm{G}}\left[\boldsymbol{e}_{h}\right]$ are computed for both cases and results are summarized in Table 4 . The effectivity index of the upper bound estimate is similar for the two meshes, and close to 1.17 ( $\rho \approx 17 \%$ ). The lower bound effectivity are not as sharp, they are close to $0.3(\rho \approx-70 \%)$. 

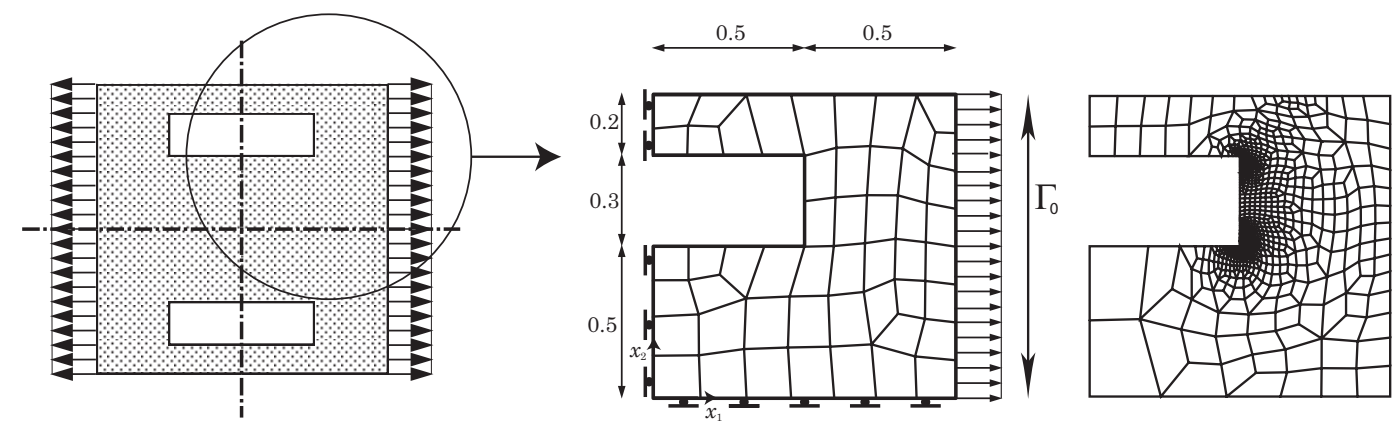

Fig. 9. Thin plate model problem and meshes with 140 d.o.f. (center) and 1970 d.o.f.(right)

Table 4

Thin plate: upper and lower bounds for $\left\|e_{h}\right\|$

\begin{tabular}{|c|cc|cc|}
\hline d.o.f. & $\left\|\boldsymbol{e}_{h}\right\|$ & $\frac{\left\|\boldsymbol{e}_{h}\right\|}{\left\|\boldsymbol{u}_{h}\right\|}$ & $\begin{array}{c}\rho\left(\mathrm{E}_{\mathrm{u}}\left[\boldsymbol{e}_{h}\right]\right) \\
\text { with } \boldsymbol{\pi}^{h}\end{array}$ & $\begin{array}{c}\rho\left(\mathrm{E}_{\mathrm{l}}^{\mathrm{G}}\left[\boldsymbol{e}_{h}\right]\right) \\
\text { with } \boldsymbol{\pi}^{h}\end{array}$ \\
\hline 140 & 0.146 & $12.8 \%$ & $17.9 \%$ & $-68.7 \%$ \\
1970 & 0.040 & $3.44 \%$ & $17.1 \%$ & $-70.1 \%$ \\
\hline
\end{tabular}
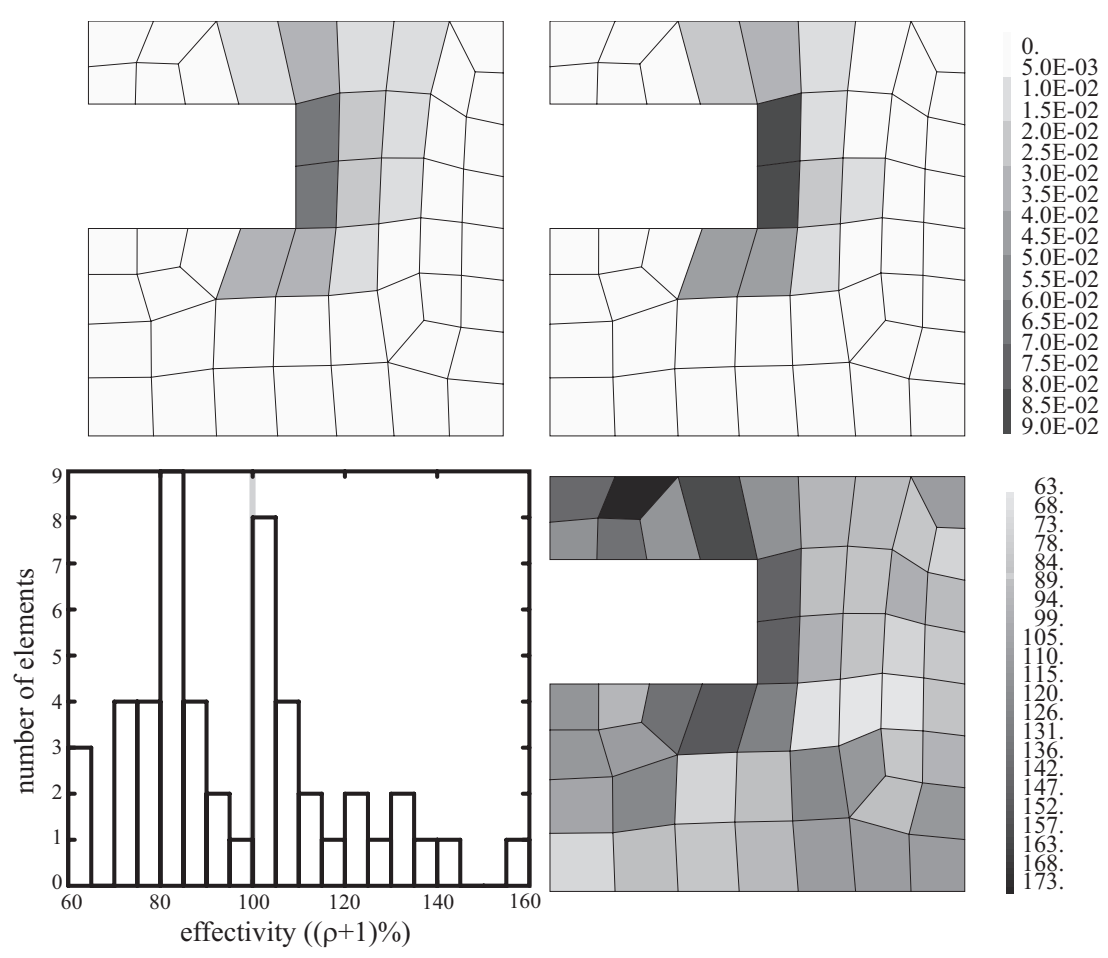

Fig. 10. Thin plate: spatial distribution of the reference error (top left), estimate $\mathrm{E}_{\mathrm{u}}\left[\boldsymbol{e}_{h}\right]$ (top right), and local distribution of the effectivity indices $(\rho+1) \%$ (bottom) for the mesh with 140 d.o.f.

Spatial distributions of error $\mathrm{E}_{\mathrm{u}}\left[\boldsymbol{e}_{h}\right]$ are displayed in Figures 10 and 11 for the uniform and adapted meshes, respectively. Note that they are computed using $\pi^{h}$ in the r.h.s of equation (12). It is worth noting that the error distributions 

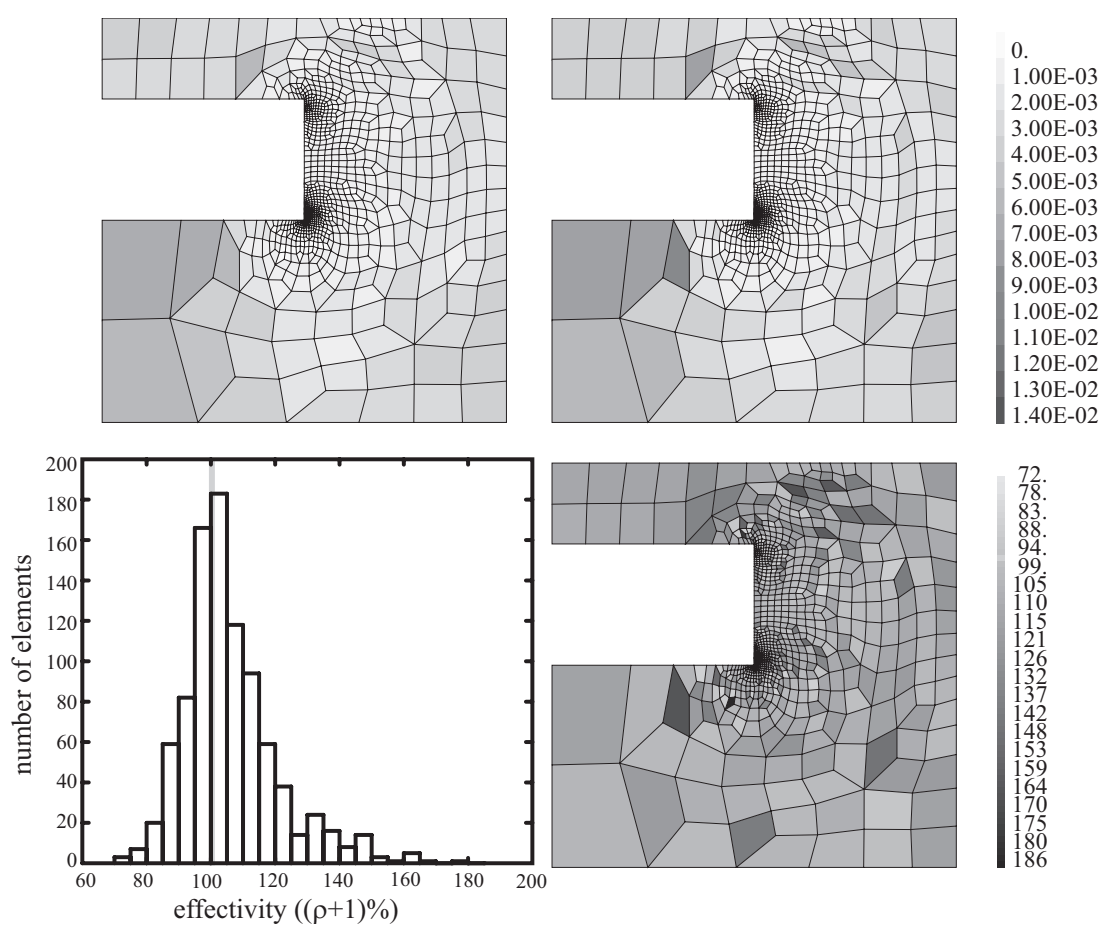

Fig. 11. Thin plate: spatial distribution of the reference error (top left), estimate $\mathrm{E}_{\mathrm{u}}\left[\boldsymbol{e}_{h}\right]$ (top right), and local distribution of the effectivity indices $(\rho+1) \%$ (bottom) for the mesh with 2588 d.o.f.

for $\mathrm{E}_{\mathrm{u}}\left[\boldsymbol{e}_{h}\right]$ are in good agreement with the reference error. The bad behavior of the local effectivity index in the first mesh, see Figure 10, is due to the fact that practically all the error is concentrated in a few relevant elements. The histogram in Figure 11 is narrow because the number of elements in the zones where the error is relevant is much higher for the second mesh.

Finally, Figure 12 shows a comparison between the proposed upper bound estimate, $\sqrt{\mathrm{E}_{\mathrm{u}}\left[\boldsymbol{e}_{h}\right]}$, the flux-free techniques proposed in $[9]\left(\mathrm{w}^{i}=1 / \sigma\right)$ and in $[8,10]\left(\mathrm{w}^{i}=\phi^{i}\right)$, and a hybrid-flux upper bound estimate, see [7,2]. The upper bound estimates are computed for a series of adapted triangular meshes. As expected all of them converge. Moreover, this is an example in which the hybrid-flux bound is sharper than the previously published flux-free upper bound estimates. In [11] the majority of the examples behave similarly. However, as already discussed the proposed flux-free bound is as sharp as the hybrid-flux one.

\subsection{Assessment of outputs of interest for a crack opening problem}

The error estimator presented in this paper is applied to the crack opening problem proposed in [21]. The specimen is described in Figure 13. Loads are a uniform pressure in the upper round cavity and a uniform normal traction 


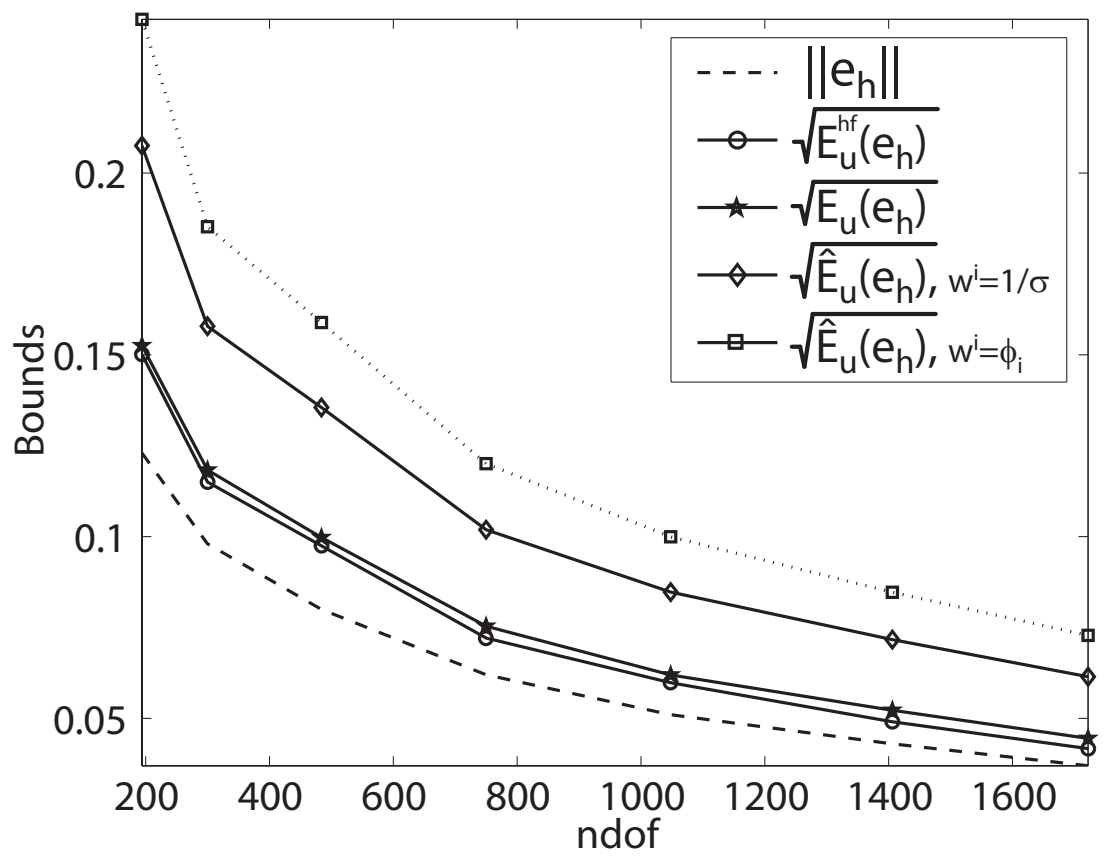

Fig. 12. Thin plate: comparison between flux-free and hybrid-flux estimates
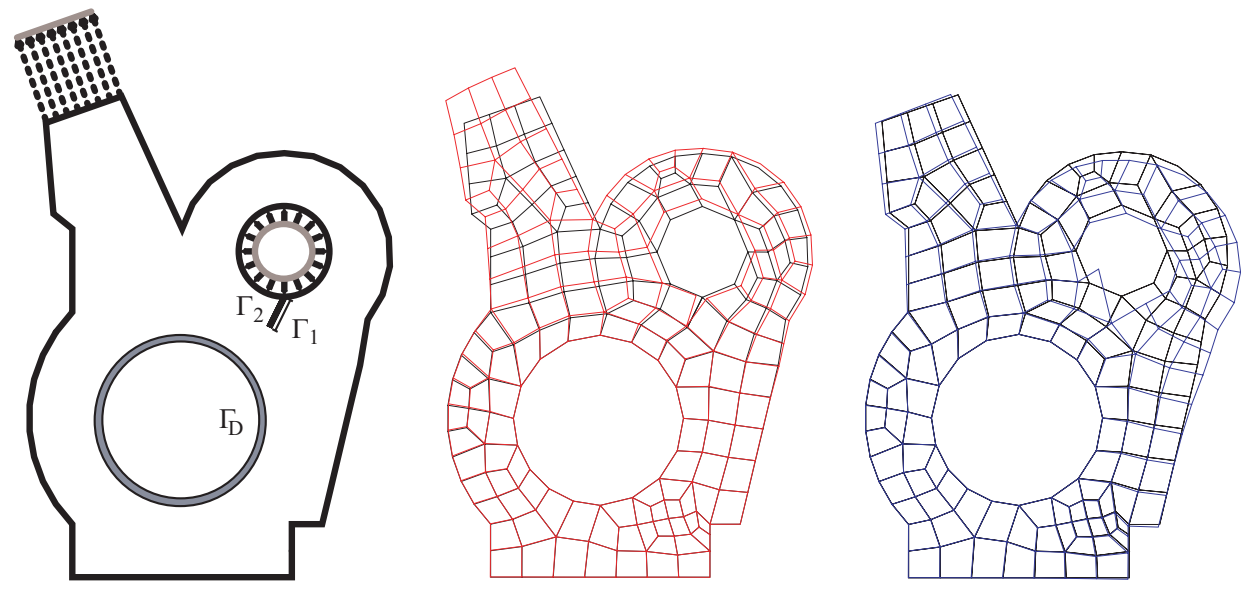

Fig. 13. Crack opening model problem (left), primal (center) and dual (right) solutions. Uniform mesh with 173 nodes (346 d.o.f)

pulling the left upper part of the specimen. Displacements are set to zero along $\Gamma_{D}$, around the centered round cavity. The edges of the crack are denoted by $\Gamma_{1}$ (right) and $\Gamma_{2}$ (left). The quantity of interest is taken as the average opening along the crack, that is,

$$
l^{\mathcal{O}}(\boldsymbol{u})=-\int_{\Gamma_{1}} \boldsymbol{u} \cdot \boldsymbol{n} d \Gamma-\int_{\Gamma_{2}} \boldsymbol{u} \cdot \boldsymbol{n} d \Gamma
$$

Note that the opposite sides of the crack, $\Gamma_{1}$ and $\Gamma_{2}$, have opposite normal unit outward vectors. Thus, $l^{\mathcal{O}}(\boldsymbol{u})$ is the average (integrated) crack opening, it is positive for opening and negative for penetration. 
Table 5

Crack opening problem: energy norm estimates and effectivity indices

\begin{tabular}{|c|ccc|ccc|}
\hline & \multicolumn{3}{|c|}{ PrimaL } & \multicolumn{3}{c|}{ DUAL } \\
\hline d.o.f & $\frac{\left\|\boldsymbol{e}_{h}\right\|}{\left\|\boldsymbol{u}_{h}\right\|}$ & $\rho\left(\mathrm{E}_{\mathrm{u}}\left[\boldsymbol{e}_{h}\right]\right)$ & $\rho\left(\mathrm{E}_{\mathrm{l}}^{\mathrm{G}}\left[\boldsymbol{e}_{h}\right]\right)$ & $\frac{\left\|\boldsymbol{\epsilon}_{h}\right\|}{\left\|\boldsymbol{\psi}_{h}\right\|}$ & $\rho\left(\mathrm{E}_{\mathrm{u}}\left[\boldsymbol{\epsilon}_{h}\right]\right)$ & $\rho\left(\mathrm{E}_{\mathrm{l}}^{\mathrm{G}}\left[\boldsymbol{\epsilon}_{h}\right]\right)$ \\
\hline 346 & $20.6 \%$ & $18.1 \%$ & $-48.8 \%$ & $61.6 \%$ & $16.4 \%$ & $-19.6 \%$ \\
1344 & $10.5 \%$ & $14.1 \%$ & $-82.9 \%$ & $25.5 \%$ & $19.1 \%$ & $-63.1 \%$ \\
\hline
\end{tabular}

Table 6

Crack opening problem: estimates for $z_{h}^{ \pm}$

\begin{tabular}{|c|ccc|ccc|}
\hline d.o.f & $\left\|\boldsymbol{z}_{h}^{+}\right\|$ & $\rho\left(\mathrm{E}_{\mathrm{u}}\left[\boldsymbol{z}_{h}^{+}\right]\right)$ & $\rho\left(\mathrm{E}_{\mathrm{l}}^{\mathrm{G}}\left[\boldsymbol{z}_{h}^{+}\right]\right)$ & $\left\|\boldsymbol{z}_{h}^{-}\right\|$ & $\rho\left(\mathrm{E}_{\mathrm{u}}\left[\boldsymbol{z}_{h}^{-}\right]\right)$ & $\rho\left(\mathrm{E}_{1}^{\mathrm{G}}\left[\boldsymbol{z}_{h}^{-}\right]\right)$ \\
\hline 346 & 0.666 & $16.7 \%$ & $-30.9 \%$ & 0.457 & $18.3 \%$ & $-49.7 \%$ \\
1344 & 0.313 & $17.2 \%$ & $-74.6 \%$ & 0.232 & $15.2 \%$ & $-84.4 \%$ \\
\hline
\end{tabular}

First, the analysis is performed with the coarse uniform mesh shown in Figure 13. Energy norm error estimates for both the primal and dual problems are summarized in Table 5. Global effectivity indices are of the same order of magnitude as in the previous example for the upper bound estimates. Although the mesh is excessively coarse and the error is large $(78 \%$ for the dual problem), the behavior of the upper bound is similar and the quality lower bound estimates is better.

Table 6 shows the energy norm estimates for the quantities $\boldsymbol{z}_{h}^{+}$and $\boldsymbol{z}_{h}^{-}$. Recall that these linear combinations of the primal and dual errors, $\boldsymbol{z}_{h}^{ \pm}=\kappa \boldsymbol{e}_{h} \pm \frac{1}{\kappa} \boldsymbol{\epsilon}_{h}$, are required to assess the error in the quantities of interest. Upper and lower bounds for the quantity of interest $l^{\mathcal{O}}\left(\boldsymbol{u}_{h}\right)$ are obtained by properly combining upper and lower bounds in the energy norm of $\boldsymbol{z}_{h}^{+}$and $\boldsymbol{z}_{h}^{-}$, see Figure 1 . Table 6 indicates that upper bound estimates of $\boldsymbol{z}_{h}^{ \pm}$present similar values of effectivity indices as in previous examples. Effectivity for lower bound estimates is again quite poor, specially for $\boldsymbol{z}_{h}^{-}$with a value of $0.08(\rho \approx-92 \%)$. Note however, that this poor lower bound effectivity does not drastically downgrade bounds of the desired functional output $l^{\mathcal{O}}\left(\boldsymbol{u}_{h}\right)$, which are computed as indicated in Figure 1 and Section 6. In fact, the obtained bounds are better than if the trivial lower bounds (equal to zero) are imposed.

In fact, for the coarse case (346 d.o.f.), when upper and lower bounds for $l^{\mathcal{O}}\left(\boldsymbol{u}_{h}\right)$ are computed from data of Table 6 , the following range is obtained: $0.141 \leq$ $l^{\mathcal{O}}\left(\boldsymbol{u}_{h}\right) \leq 0.299$. Note that the coarse mesh estimate is $l^{\mathcal{O}}\left(\boldsymbol{u}_{H}\right)=0.161$ and the reference one is $l^{\mathcal{O}}\left(\boldsymbol{u}_{h}\right)=0.220$. In this case, the mesh is very inaccurate for the desired quantity of interest because the error of $l^{\mathcal{O}}\left(\boldsymbol{u}_{H}\right)$ is $26.8 \%$ with respect to the reference one. Nevertheless, the estimate $0.220 \pm 0.079$ is a valuable information.

The same analysis is performed with the mesh (1344 d.o.f.) shown in Figure 
Fig. 14. Crack opening: heuristically adapted mesh with 672 nodes (1344 d.o.f.)

14. This mesh is uniformly densified and heuristically adapted concentrating elements in the neighborhood of the crack tip. As Table 6 shows, the energy errors are improved (10.5\% and $26.4 \%$ for primal and dual problems) and the error of $l^{\mathcal{O}}\left(\boldsymbol{u}_{H}\right)$ is now $5.11 \%$ with respect to the reference one. Thus the output gap is reduced since $0.200 \leq l^{\mathcal{O}}\left(\boldsymbol{u}_{h}\right) \leq 0.249$ and the estimate is sharper $0.225 \pm 0.025$.

Figure 15 shows the spatial distribution of the element-by-element contributions to $l^{\mathcal{O}}\left(\boldsymbol{e}_{h}\right)$. That is, the local values for $a_{k}\left(\boldsymbol{e}_{h}, \boldsymbol{\epsilon}_{h}\right)$ in every element $\Omega_{k}$ of the mesh are plotted. Note that this is not an estimate but the actual reference values. These local contributions may be either positive or negative. In order to better depict the areas that contribute to the error the distribution is represented by both the absolute value and the sign of the local contributions. The distribution of the absolute value shows that the main contributions to the error in the quantity of interest are related to elements in the neighborhood of the crack tip. Such a distribution of the error could guide an adaptive process.

The estimated spatial distribution of the error in the output of interest is also shown in Figure 15. That is, the estimated values $\tilde{\boldsymbol{e}}$ and $\tilde{\boldsymbol{\epsilon}}$, see for instance step 2 in Figure 5, are used to evaluate $a_{k}(\tilde{\boldsymbol{e}}, \tilde{\boldsymbol{\epsilon}})$ in every element $\Omega_{k}$ of the mesh. The similarity of these distributions demonstrates the good agreement between the true and the estimated error distributions. Therefore, the introduced error estimators may be fairly used in a goal-oriented adaptive analysis.

\subsection{D mechanical problem}

As previously observed, this approach easily accommodates a 3D analysis. Note that the modifications in code for the 3D analysis showed here were 

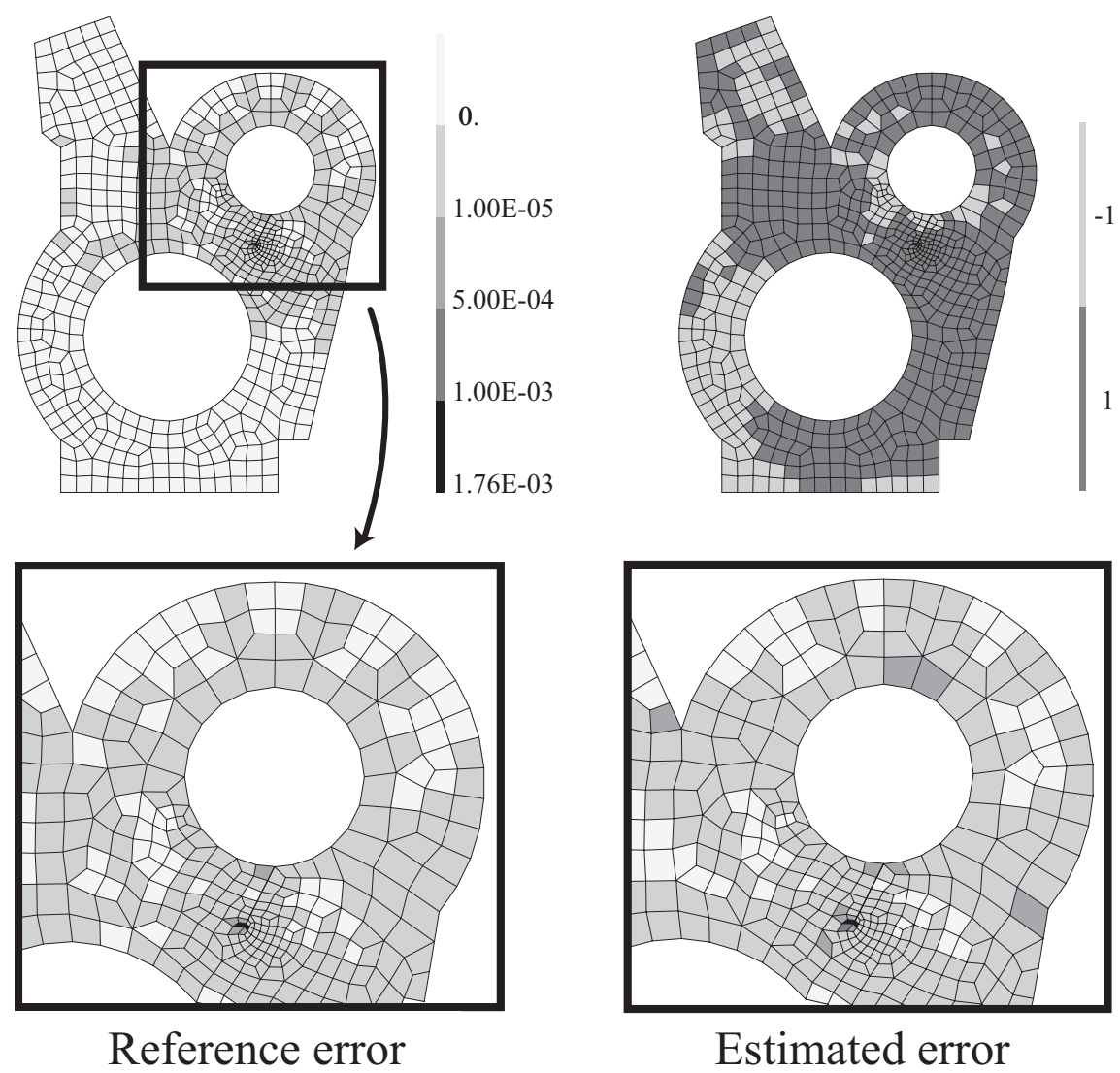

Estimated error

Fig. 15. Spatial distribution of the absolute value of the local contributions to $l^{\mathcal{O}}\left(\boldsymbol{e}_{h}\right)$ (upper-left and zoom into the relevant zones). The zoom boxes compare the reference error distribution with the estimated error distribution. The upper-right plot describes the sign of these local contributions for the reference error distribution.

developed in four hours starting from the 2D implementation. Moreover, in $3 \mathrm{D}$ the conclusions drawn in [11] from a computational cost point of view are more critical.

The geometry of the problem is inspired in an arch structure proposed in [23]. The structure is casted in the bottom bases and loaded with a uniform pressure on one lateral side, see Figure 16. This figure also shows the mesh of 174 quadratic 10-noded tetrahedra used for the analysis. The error assessment is also performed on the mesh shown in Figure 17, which has been heuristically adapted by refining the elements along the loaded lateral side where stresses are larger. This adapted mesh contains 695 tetrahedra and 1495 nodes.

In this example, we compute the estimates $\mathrm{E}_{\mathrm{u}}\left[\boldsymbol{e}_{h}\right]$, and $\mathrm{E}_{\mathrm{l}}^{\mathrm{G}}\left[\boldsymbol{e}_{h}\right]$ introduced in this paper using the projection $\pi^{h}$ for the r.h.s. term of the residual equation, as described in Section 7.1. This estimate is compared with $\hat{\mathrm{E}}_{\mathrm{u}}\left[\boldsymbol{e}_{h}\right]$ described in $[8,10]\left(\right.$ with $\left.\mathrm{w}^{i}=\phi^{i}\right)$.

The reference mesh is obtained dividing each tetrahedron in 8 tetrahedra. 

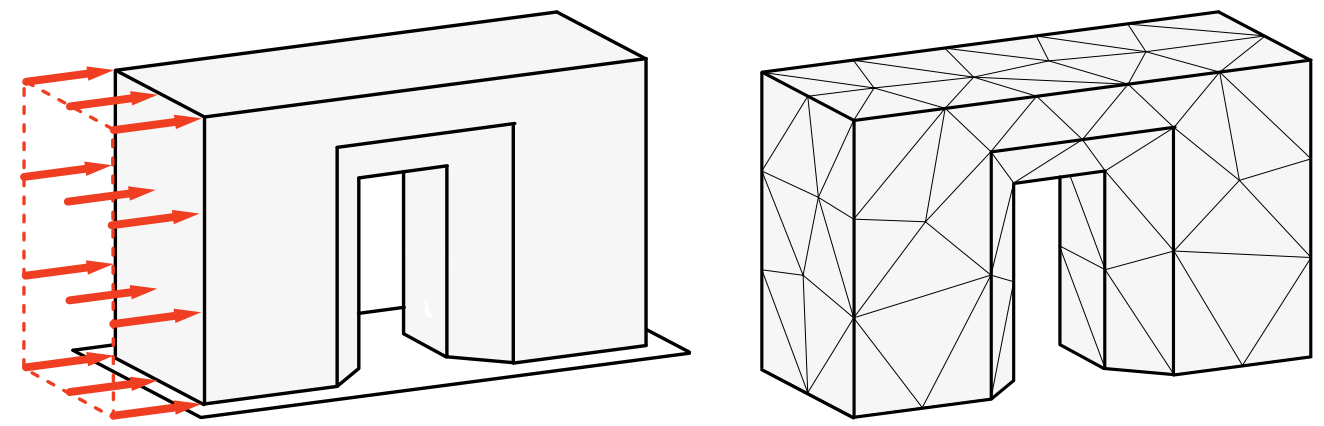

Fig. 16. 3D model problem (left) and uniform mesh (right) with 174 elements and 401 nodes

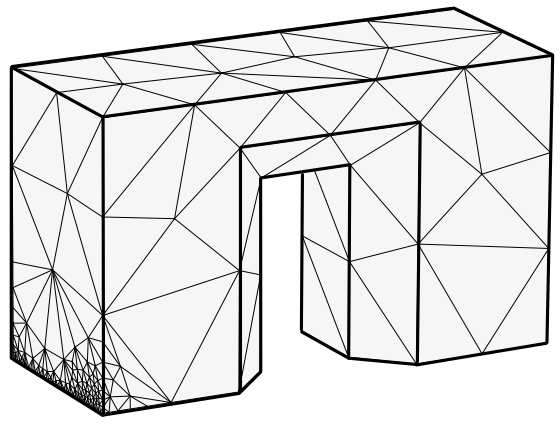

Fig. 17. 3D adapted mesh with 695 elements and 1495 nodes

Table 7

3D upper and lower bounds for $\left\|\boldsymbol{e}_{h}\right\|$

\begin{tabular}{|cc|cc|ccc|}
\hline Mesh & d.o.f. & $\left\|\boldsymbol{e}_{h}\right\|$ & $\frac{\left\|\boldsymbol{e}_{h}\right\|}{\left\|\boldsymbol{u}_{h}\right\|}$ & $\rho\left(\mathrm{E}_{\mathrm{u}}\left[\boldsymbol{e}_{h}\right]\right)$ & $\rho\left(\mathrm{E}_{\mathrm{l}}^{\mathrm{G}}\left[\boldsymbol{e}_{h}\right]\right)$ & $\rho\left(\hat{\mathrm{E}}_{\mathrm{u}}\left[\boldsymbol{e}_{h}\right]\right)$ \\
Uniform & 1203 & 4.13 & $22.0 \%$ & $8.18 \%$ & $-8.56 \%$ & $53.7 \%$ \\
Adapted & 4485 & 3.63 & $19.2 \%$ & $9.67 \%$ & $-14.4 \%$ & $58.6 \%$ \\
\hline
\end{tabular}

Table 7 shows that the estimate proposed here is one order of magnitude sharper than the one introduced in $[8,10]$. Note that lower bound estimates present similar effectivities.

Surprisingly, comparing results for the uniform mesh with 1203 d.o.f and the adapted mesh with 4485 d.o.f., the reference error is reduced only from $22.0 \%$ to $19.2 \%$. This is due to the fact that the first mesh is too coarse and the corresponding reference mesh is not accurate enough. Using this reference mesh the norm of the reference error is 4.13 , the exact error is however much larger. A finer reference mesh is built up by splitting each element into 64 tetrahedra (instead of 8). Due to the size of the problem, for this very fine reference mesh, the reference error can be estimated but it cannot be computed. The obtained values for the upper bound estimate $\mathrm{E}_{\mathrm{u}}\left[\boldsymbol{e}_{h}\right]$ and the lower bound estimate $\mathrm{E}_{1}^{\mathrm{G}}\left[\boldsymbol{e}_{h}\right]$ are 4.34 and 5.24 respectively. Thus the exact error is approximately 5 (probably larger). That means that for the uniform mesh the error is closer $27 \%$ than $22 \%$. 


\section{Concluding remarks}

This paper introduces a new technique to compute upper and lower bounds for functional outputs. As previously proposed subdomain-based approaches, the implementation of the proposed method is less cumbersome compared to hybrid-flux estimators where flux equilibration algorithms must be implemented. Moreover, it can be used in the mechanical case for linear elements and the accuracy of the results (sharpness of the upper bound) are drastically improved compared to other flux-free techniques. In fact, the observed accuracy is at least comparable to hybrid-flux methods.

The resulting estimates yield guaranteed (and sharp) upper bounds of the reference error. A simple postprocessing yields lower bounds of the error with a little extra computational cost. Lower bounds of the energy error are useful to sharpen upper and lower bounds of the error in quantities of interest.

The local problems that have to be solved in this context are flux-free, that is no flux equilibration is required. The flux-free property is specially significant when compared with the standard residual type error estimators (hybrid-flux approach). The local boundary conditions for the local problems in the standard estimators require flux equilibration and result in costly computations and complex programming, especially in 3D.

The distribution of the local contributions to the error are also accurately estimated, both for the energy norm of the error and for the error measured using some functional output. These estimates are therefore well suited to guide goal-oriented adaptive procedures.

\section{References}

[1] M. Paraschivoiu, J. Peraire, A. T. Patera, A posteriori finite element bounds for linear-functional outputs of elliptic partial differential equations, Comput. Methods Appl. Mech. Eng. 150 (1-4) (1997) 289-312, symposium on Advances in Computational Mechanics, Vol. 2 (Austin, TX, 1997).

[2] M. Ainsworth, J. T. Oden, A posteriori error estimation in finite element analysis, John Wiley \& Sons, Chichester, 2000.

[3] J. T. Oden, S. Prudhomme, Goal-oriented error estimation and adaptivity for the finite element method, Comput. Math. Appl. 41 (5-6) (2001) 735-756.

[4] J. Bonet, A. Huerta, J. Peraire, The efficient computation of bounds for functionals of finite element solutions in large strain elasticity, Comput. Methods Appl. Mech. Engrg. 191 (43) (2002) 4807-4826. 
[5] A. M. Sauer-Budge, J. Bonet, A. Huerta, J. Peraire, Computing bounds for linear functionals of exact weak solutions to Poisson's equation, SIAM J. Numer. Anal. 42 (4) (2004) 1610-1630.

[6] N. Pares, J. Bonet, A. Huerta, J. Peraire, The computation of bounds for linearfunctional outputs of weak solutions to the two-dimensional elasticity equations, Comput. Methods Appl. Mech. Eng. 194.

[7] P. Ladevèze, D. Leguillon, Error estimate procedure in the finite element method and applications, SIAM J. Numer. Anal. 20 (3) (1983) 485-509.

[8] C. Carstensen, S. A. Funken, Fully reliable localized error control in the FEM, SIAM J. Sci. Comput. 21 (4) (1999/00) 1465-1484 (electronic).

[9] L. Machiels, Y. Maday, A. T. Patera, A "flux-free" nodal Neumann subproblem approach to output bounds for partial differential equations, C. R. Acad. Sci. Paris Sér. I Math. 330 (3) (2000) 249-254.

[10] P. Morin, R. H. Nochetto, K. G. Siebert, Local problems on stars: a posteriori error estimators, convergence, and performance, Math. Comp. 72 (243) (2003) 1067-1097.

[11] H.-W. Choi, M. Paraschivoiu, Adaptive computations of a posteriori finite element output bounds: a comparison of the "hybrid-flux" approach and the "flux-free" approach, Comput. Methods Appl. Mech. Eng. 193 (36-38) (2004) 4001-4033.

[12] Y. Maday, A. T. Patera, J. Peraire, A general formulation for a posteriori bounds for output functionals of partial differential equations; application to the eigenvalue problem, C. R. Acad. Sci. Paris Sér. I Math. 328 (9) (1999) $823-828$.

[13] S. Prudhomme, J. T. Oden, On goal-oriented error estimation for elliptic problems: application to the control of pointwise errors, Comput. Methods Appl. Mech. Eng. 176 (1-4) (1999) 313-331, new advances in computational methods (Cachan, 1997).

[14] A. T. Patera, J. Peraire, A general Lagrangian formulation for the computation of a posteriori finite element bounds, in: Error estimation and adaptive discretization methods in computational fluid dynamics, Vol. 25 of Lect. Notes Comput. Sci. Eng., Springer, Berlin, 2003, pp. 159-206.

[15] I. Babuška, W. C. Rheinboldt, Error estimates for adaptive finite element computations, SIAM J. Numer. Anal. 15 (4) (1978) 736-754.

[16] P. Díez, N. Parés, A. Huerta, Recovering lower bounds of the error by postprocessing implicit residual a posteriori error estimates, Internat. J. Numer. Methods Engrg. 56 (10) (2003) 1465-1488.

[17] A. Huerta, P. Díez, Error estimation including pollution assessment for nonlinear finite element analysis, Comput. Methods Appl. Mech. Eng. 181 (1-3) (2000) 21-41. 
[18] S. C. Brenner, L. R. Scott, The mathematical theory of finite element methods, 2nd Edition, Vol. 15 of Texts in Applied Mathematics, Springer-Verlag, New York, 2002.

[19] R. E. Bank, A. Weiser, Some a posteriori error estimators for elliptic partial differential equations, Math. Comp. 44 (170) (1985) 283-301.

[20] M. Ainsworth, J. T. Oden, A unified approach to a posteriori error estimation using element residual methods, Numer. Math. 65 (1) (1993) 23-50.

[21] I. Babuška, T. Strouboulis, C. S. Upadhyay, S. K. Gangaraj, K. Copps, Validation of a posteriori error estimators by numerical approach, Int. J. Numer. Methods Eng. 37 (7) (1994) 1073-1123.

[22] J. Peraire, A. T. Patera, Bounds for linear-functional outputs of coercive partial differential equations: local indicators and adaptive refinement, in: Advances in adaptive computational methods in mechanics (Cachan, 1997), Vol. 47 of Stud. Appl. Mech., Elsevier, Amsterdam, 1998, pp. 199-216.

[23] E. Florentin, L. Gallimard, J. P. Pelle, Evaluation of the local quality of stresses in 3D finite element analysis, Comput. Methods Appl. Mech. Eng. 191 (39-40) (2002) 4441-4457. 